\title{
Central summation in the quasicontinuum method
}

\author{
L.A.A. Beex, R.H.J. Peerlings*, M.G.D. Geers \\ Department of Mechanical Engineering, Eindhoven University of Technology \\ P.O. Box 513, 5600 MB Eindhoven, The Netherlands
}

\begin{abstract}
The quasicontinuum (QC) method [Tadmor, E.B., Phillips, R., Ortiz, M., 1996, Mixed atomistics and continuum models of deformation in solids, Langmuir, Vol. 12, 4529-4534] is a multiscale methodology to significantly reduce the computational cost of atomistic simulations. The method ensures an accurate incorporation of small-scale atomistic effects in large-scale models. It essentially consists of an interpolation of the displacements of large numbers of atoms between representative atoms (repatoms) and an estimation of the total potential energy of the atomistic lattice by a so-called summation (or sampling) rule. In this paper a novel energy-based summation rule is presented for the QC method that allows for a seamless coupling between coarse domains and fully resolved domains. In the presented summation rule only the repatoms are used in combination with one extra sampling atom in the center of each interpolation triangle. The presented summation rule is therefore straightforward and computationally efficient. The performance of the proposed summation rule is evaluated for a number of two-dimensional and three-dimensional multiscale atomistic test problems.
\end{abstract}

Key words: quasicontinuum method, summation rule, summation, multiscale, atomistic model, coarse graining, numerical algorithms

\footnotetext{
${ }^{*}$ Corresponding author

Address: R.H.J. Peerlings, Department of Mechanical Engineering, Eindhoven University of Technology, P.O. Box 513, 5600 MB Eindhoven, The Netherlands.

Email: r.h.j.peerlings@tue.nl
} 


\section{Introduction}

The quasicontinuum (QC) method (Tadmor et al., 1996a,b) is a multiscale approach that reduces the computational cost of atomistic lattice computations. The QC method directly takes atomistic effects into account in large-scale models. In different studies the methodology has been used to investigate vacancies (Shimokawa et al., 2004; Gavini et al., 2007), dislocations (Tadmor et al., 1996a; Hayes et al., 2004), cracks (Miller et al., 1998), grain boundaries (Miller et al., 1998; Shimokawa et al., 2004), nanovoids (Marian et al., 2008) and carbon nanotubes (Park et al., 2010). A substantial part of the QC studies has focused on nanonindentation (Tadmor et al., 1996b, 1999; Knap and Ortiz, 2001, 2003; Shenoy et al., 1999; Picu, 2000; Miller and Rodney, 2002; Eidel and Stukowski, 2009; Kwon et al., 2009). Furthermore, extensions of the method have been developed to deal with phase transformations (Dobson et al., 2007), density functional theory (Hayes et al., 2004; Gavini et al., 2007), finite temperatures (Kulkarni et al., 2008; Marian et al., 2010) and long-range interatomic interactions (Zhang and Gunzburger, 2010). Although the QC method was so far mainly used for atomistic lattice models, it may also be extended to microstructural lattice models, as shown by Beex et al. (2011). Reviews of different QC methods and related multiscale methods are presented by Miller and Tadmor (2002, 2009) and Curtin and Miller (2003).

The QC method uses two approximations to reduce the computational cost of full atomistic computations. First, only a small number of atoms (repatoms) is selected to represent the displacements of all atoms in the entire lattice. The repatoms constrain the displacements of the atoms in between them by means of interpolation. The displacement components of the repatoms are the only remaining degrees of freedom (DOFs) of the interpolated atomistic lattice. In regions where the displacement fluctuations are small, it suffices to select repatoms far away from each other, while in regions with large displacement fluctuations every atom constitutes a repatom so that the exact atomistic model is recovered in these regions of interest.

The second approximation introduced in the QC method is the selection of only a small number of atoms (to which we will refer as sampling atoms) to estimate the total potential energy of the atomistic lattice, by a so-called summation rule, instead of visiting all atoms of the crystal to compute it exactly. The sampling atoms are used to estimate the site-energies of the atoms in their vicinity. To ensure an accurate estimation of the total potential 
energy, it is important that the selection of sampling atoms is made carefully taking into account the triangulation used for the interpolation. If this is not the case, inaccuracies or even zero-energy modes may occur (Knap and Ortiz, 2001).

Based on the type of summation rule used, two classes of QC methodologies are distinguished. In local-nonlocal QC methodologies (Tadmor et al., 1996a,b; Miller et al., 1998; Shenoy et al., 1999) the site-energies of the sampling atoms in the interpolated domain are locally computed using the Cauchy-Born rule and the site-energies of the sampling atoms in fully resolved domains are computed in a nonlocal manner. This leads to an internal interface between both domains at which an error is introduced due to so-called ghost-forces. To ensure that the ghost-forces at the interface have no significant influence at the solution in the center of the region of interest, the fully resolved region must be relatively large. As a result the interface is located relatively far away from the center of the region of interest. This increases the computational cost. Corrective procedures have been developed (Shenoy et al., 1999), but they come with additional assumptions.

The second class of summation rules is that of fully nonlocal cluster summation rules (Knap and Ortiz, 2001; Eidel and Stukowski, 2009). Fully nonlocal cluster summation rules treat the coarse domain and the fully resolved region identically. For this reason no internal interface occurs and both domains can be seamlessly coupled to each other. Luskin and Ortner (2008) however have shown that even for large clusters this summation rule gives a poor estimate of the total potential energy.

In addition to the above two established classes, new summation rules have recently been proposed. In the studies of Zhang and Gunzburger (2010) and Gunzburger and Zhang (2010) the selection of sampling atoms is based on numerical quadrature as used in the finite element (FE) method. The atoms located at (or nearby) the Gauss points are selected as sampling atoms. The obtained results are more accurate than those obtained with the cluster QC variant for a one-dimensional chain of atoms. This summation rule has so far only been applied on one-dimensional atomistic chains. The one-dimensional quadrature-type summation rule has been recently compared to the nodal variant of the cluster summation rule by Iyer and Gavini (2011).

In our previous study (Beex et al., 2011), an exact, fully nonlocal summation rule is proposed for structural lattice models in which only nearest neighbor interactions exist. It is based on the proper understanding of how the total potential energy is related to the triangulation. As a result, the 
computed total potential energy is not an estimate but exact for the given interpolation and the remaining error is only due to the interpolation itself. However, if the same summation rule is to be used for atomistic crystals including next-to-nearest neighbor interactions, the number of sampling atoms becomes prohibitively large so that the method becomes computationally inefficient.

The aim of this paper is to develop a new summation rule, inspired by the one of Beex et al. (2011), for two-dimensional and three-dimensional atomistic crystals. It ensures a seamless coupling between coarse domains and fully resolved domains as a result of the nonlocal site-energy computation of the sampling atoms in coarse domains as well as in fully resolved domains. The absence of an internal interface increases the computational efficiency because no updating of interface corrections is necessary if fully resolved regions are adapted or moved through the coarse domain. This leads to a simple and unified multiscale QC approach, like the cluster QC method. However, since it is based on the exact recovery of the total potential energy, a better estimate of the potential energy is obtained than for the cluster QC method.

The computational cost of the energy-based summation rule is low, because it selects the repatoms as sampling atoms plus one sampling atom in the center of each triangle (or tetrahedron) of the interpolation. The repatoms only sample themselves (i.e. have a weight factor of one) so that in the fully resolved region the exact atomistic model is recovered. One sampling atom near the center of each interpolation triangle (or tetrahedron for three-dimensional crystals) is used to sample the remaining atoms. Since the focus of the summation rule is on the center of the triangles and tetrahedra we refer to the new summation rule as the central summation rule.

The outline of this paper is as follows. In Section 2 the QC method is briefly reviewed and in Section 3 the existing summation rules are explained in somewhat more detail. In Section 4 the exact relation between the total potential energy and the interpolation triangulation is discussed. In Section 5 the central summation rule is derived. The results of the central summation rule are discussed in Section 6 for a two-dimensional atomistic lattice, a simplified two-dimensional atomistic lattice and a three-dimensional atomistic lattice. Furthermore, a comparison is made with the results of the cluster QC method (Knap and Ortiz, 2001) as improved by Eidel and Stukowski (2009) for the two-dimensional atomistic lattice. In all numerical tests, the solution near a vacancy at the center of the fully resolved domain is investigated and compared. Finally, conclusions are presented in Section 7. 


\section{The quasicontinuum method}

The descriptions of the direct atomistic lattice problem and quasicontinuum approximation in this section are formulated in terms of potential energy and minimization thereof. The central summation rule formulated below is energy-based, as those of Eidel and Stukowski (2009) and Beex et al. (2011). For force-based formulations the study of Knap and Ortiz (2001) may form a starting point.

\subsection{Problem formulation}

The total potential energy of an atomistic lattice is the sum of the internal and external potential energy, where the internal potential energy is assumed to be the sum of the site-energies of all atoms of the lattice:

$$
E_{t o t}(\mathbf{u})=\sum_{i=1}^{n} E_{i}(\mathbf{u})-\mathbf{f}_{e x t}^{T} \mathbf{u} .
$$

In this expression the total potential energy is denoted by $E_{t o t}$ and the siteenergy of atom $i$ by $E_{i}$. The $n$ atoms of the lattice are stored in index set $N=\{1, . ., n\}$. The external potential energy $E_{\text {ext }}$ is a function of the components of the externally applied forces, stored in column matrix, $\mathbf{f}_{\text {ext }}$, and the displacement components of all atoms which are stored in column matrix $\mathbf{u}$. Columns $\mathbf{f}_{e x t}$ and $\mathbf{u}$ are both of length $n d$, in which $d$ symbolizes the number of spatial dimensions. The site-energy $E_{i}$ is formulated as a function of the interatomic potential energies, $E_{i j}$, between atom $i$ and its neighbors $j$ within a cut-off radius, which are stored in subset $B_{i}\left(B_{i} \subseteq N\right)$ :

$$
E_{i}(\mathbf{u})=\frac{1}{2} \sum_{j \in B_{i}} E_{i j}(\mathbf{u}) .
$$

Half of the interatomic potential energy $E_{i j}$ between atom $i$ and neighboring atom $j$ is thus assigned to atom $i$ and the other half to atom $j$. For the interatomic potential energy $E_{i j}$ the Lennard-Jones potential (Lennard-Jones, 1937) can be used, as was done by Knap and Ortiz (2001, 2003), or the embedded atom method (EAM), as done by (Shimokawa et al., 2004; Miller and Tadmor, 2009). As a result of using the EAM, an additional contribution must be added to the site-energy $E_{i}$ that corrects for the electron density. 
Now the total potential energy $E_{\text {tot }}$ has been established, it can be minimized with respect to the degrees of freedom (DOFs) of the system, i.e. the displacement components of all atoms, stored in column matrix $\mathbf{u}$ :

$$
\min _{\mathbf{u}} E_{t o t}(\mathbf{u})
$$

The minimum of $E_{t o t}$ can be determined using classical variational principles. This leads to the following expression:

$$
\delta E_{t o t}(\mathbf{u})=\delta \mathbf{u}^{T} \mathbf{f}(\mathbf{u})=0 \quad \forall \delta \mathbf{u}
$$

where $\mathbf{f}(\mathbf{u})$ is a column matrix containing conservative forces; it is of length $n d$. Arbitrary kinematically admissible variations of $\mathbf{u}$ are represented here by $\delta \mathbf{u}$. With respect to the formulation of Eq. (1), it is convenient to assemble the force column matrix, $\mathbf{f}(\mathbf{u})$, from contributions $\mathbf{f}_{i}$ of each atom according to:

$$
\mathbf{f}(\mathbf{u})=-\mathbf{f}_{e x t}+\sum_{i=1}^{n} \mathbf{f}_{i}(\mathbf{u})
$$

where

$$
\left(f_{i}\right)_{p}=\frac{\partial E_{i}}{\partial u_{p}}
$$

and $p$ runs over all $n d$ displacement components present in column matrix u.

A standard Newton-Raphson process (using a first-order Taylor expansion) can now be used to solve Eq. (4):

$$
\delta \mathbf{u}^{T}\left(\mathbf{f}\left(\mathbf{u}^{*}\right)+\mathbf{K}\left(\mathbf{u}^{*}\right) d \mathbf{u}\right)=0 \quad \forall \delta \mathbf{u}
$$

where $\mathbf{u}^{*}$ are the displacement components of the previous iteration and $d \mathbf{u}$ is the correction to these displacement components computed in the present 
iteration. The overall stiffness matrix in Eq. (7) is represented by $\mathbf{K}(\mathbf{u})$. The elements of the stiffness matrix can be established as follows:

$$
K_{p q}=\frac{\partial^{2} E_{t o t}}{\partial u_{q} \partial u_{p}}=\sum_{i=1}^{n} \frac{\partial^{2} E_{i}}{\partial u_{q} \partial u_{p}}
$$

where $p$ and $q$ run over all $n d$ displacement components. The stiffness matrix is thus of size $n d \times n d$ and is symmetric. Similarly to the force column matrix, the stiffness matrix $\mathbf{K}$ can also be assembled by contributions $K_{i}$ of each atom:

$$
\mathbf{K}(\mathbf{u})=\sum_{i=1}^{n} \mathbf{K}_{i}(\mathbf{u})
$$

where

$$
\left(K_{i}\right)_{p q}=\frac{\partial^{2} E_{i}}{\partial u_{q} \partial u_{p}} .
$$

In order to solve Eq. (7), Neumann and Dirichlet boundary conditions must be incorporated, i.e. by partitioning the system. A locally stable configuration of the atomistic lattice is obtained if the part of the resulting overall stiffness matrix is positive definite.

\subsection{Interpolation}

The first approximation used by the QC method aims to reduce the large number of DOFs contained in $\mathbf{u}$ by interpolation. Interpolation is imposed by selecting a small number of $r$ repatoms which are used to represent the displacements of all atoms of the lattice. The index set $R$ of repatoms is therefore a subset of $N(R \subseteq N)$. The domain is triangulated such that the repatoms form the corners of the triangles (or tetrahedra in three dimensions). The displacements of the atoms within a triangle are interpolated (and thus constrained) between those of the repatoms at the corners of the triangle. Linear interpolation functions are generally used to express the displacement components of all atoms, stored in column $\mathbf{u}$, as a function of the displacement components of the repatoms, stored in column $\mathbf{u}_{r}$ : 


$$
\mathbf{u}=\Psi \mathbf{u}_{r}
$$

In this equation $\Psi$ represents the condensation matrix, with size $n d \times r d$, in which the interpolation function values at the locations of all $n$ atoms are gathered.

At this point, the total potential energy of the interpolated atomistic crystal only depends on the displacements of the repatoms instead of the displacements of all atoms. In regions where displacement fluctuations are small, relatively large distances between the repatoms are allowed, but in regions with large displacement fluctuations the repatoms must be located close to each other. The minimum distance equals one lattice spacing and adopting this distance as the grid size results in a fully resolved region. By correctly selecting the repatoms for a specific problem, i.e. by creating a proper triangulation, the total potential energy of the condensed (triangulated) lattice, $E_{t o t}^{r}$, is assumed to be a good approximation of the total potential energy of the full lattice, $E_{t o t}$ :

$$
E_{t o t}(\mathbf{u}) \approx E_{t o t}^{r}\left(\Psi \mathbf{u}_{r}\right)=\sum_{i=1}^{n} E_{i}\left(\Psi \mathbf{u}_{r}\right)-\mathbf{f}_{e x t}^{T} \Psi \mathbf{u}_{r}
$$

Since the DOFs of the condensed lattice are now the displacement components of the repatoms, present in $\mathbf{u}_{r}$, the minimization of $E_{\text {tot }}^{r}$ must also be performed with respect to this reduced set of DOFs:

$$
\min _{\mathbf{u}_{r}} E_{t o t}^{r}\left(\mathbf{\Psi} \mathbf{u}_{r}\right)
$$

Following the same reasoning which led to Eq. (7), now results in the following linear iterative problem:

$$
\delta \mathbf{u}_{r}^{T}\left(\boldsymbol{\Psi}^{T} \mathbf{f}\left(\boldsymbol{\Psi} \mathbf{u}_{r}\right)+\boldsymbol{\Psi}^{T} \mathbf{K}\left(\boldsymbol{\Psi} \mathbf{u}_{r}\right) \boldsymbol{\Psi} d \mathbf{u}_{r}\right)=0 \quad \forall \delta \mathbf{u}_{r}
$$

in which $\Psi^{T} \mathbf{f}$ is a condensed force column which we will denote $\mathbf{f}^{r}$, and $\boldsymbol{\Psi}^{T} \mathbf{K} \boldsymbol{\Psi}$ the condensed stiffness matrix, $\mathbf{K}^{r}$. They are of size $r d \times 1$ and 
$r d \times r d$ respectively and can be constructed according to the following expressions:

$$
\begin{aligned}
& \mathbf{f}^{r}\left(\mathbf{u}_{r}\right)=-\Psi^{T} \mathbf{f}_{e x t}+\sum_{i=1}^{n} \Psi^{T} \mathbf{f}_{i}\left(\Psi \mathbf{u}_{r}\right) \\
& \mathbf{K}^{r}\left(\mathbf{u}_{r}\right)=\sum_{i=1}^{n} \boldsymbol{\Psi}^{T} \mathbf{K}_{i}\left(\boldsymbol{\Psi} \mathbf{u}_{r}\right) \Psi
\end{aligned}
$$

where $\mathbf{f}_{i}$ and $\mathbf{K}_{i}$ represent the force column and stiffness matrix contributions associated with atom $i$ and their components are given by Eq. (6) and (10). Since the condensed stiffness matrix is substantially smaller than the original stiffness matrix $(r \ll n)$, the condensed equation in Eq. (14) is computationally more efficient to solve than the uncondensed equation (Eq. (7)).

\subsection{Summation}

Although the number of scalar equations associated with the interpolated lattice (resulting from Eq. (14)) is significantly smaller than the number of scalar equations of the full lattice (Eq. (7)), the assembly of the condensed force column and stiffness matrix still remains computationally expensive, since all $n$ atoms must be visited according to Eq. (15) and Eq. (16). To overcome this, the QC method proposes to select only a small number of $s$ atoms to estimate the total potential energy, instead of visiting all $n$ atoms to exactly determine the total potential energy. These so-called sampling atoms (stored in index set $S$ ) are used to sample the site-energies of their surrounding atoms and are selected from set $N$ containing all atoms, i.e. $S \subseteq$ $N$.

The total energy of the sampled, condensed lattice, $E_{t o t}^{r s}$, ishould be a good approximation of the total energy of the condensed lattice, $E_{t o t}^{r}$. How accurate this approximation is depends on how the sampling atoms are selected. For the summation rule proposed by Beex et al. (2011), the total potential energy of the sampled, condensed lattice is exact to the total potential energy of the condensed lattice, however at the cost of accounting for many sampling atoms. In general, we have for $E_{t o t}^{r s}$ :

$$
E_{\text {tot }}^{r}\left(\mathbf{\Psi} \mathbf{u}_{r}\right) \approx E_{\text {tot }}^{r s}\left(\mathbf{\Psi} \mathbf{u}_{r}\right)=\sum_{i \in S} w_{i} E_{i}\left(\boldsymbol{\Psi} \mathbf{u}_{r}\right)-\sum_{i \in S} w_{i} \mathbf{f}_{e x t, i}^{T} \Psi \mathbf{u}_{r},
$$


in which the site-energy of atom $i, E_{i}$, represents the site-energies of the atoms in the vicinity of atom $i$, including atom $i$ itself. The number of atoms it represents is accounted for in the weight factor $w_{i}$. Column matrix $\mathbf{f}_{e x t, i}$ only contains the external forces acting on atom $i$. Although it is of size $n d \times 1$, it thus only has non-zero entries on the locations that are related to atom $i$.

Since the DOFs of the lattice are still the displacement components of the repatoms, the total potential energy minimisation must still be performed with the respect to $\mathbf{u}_{r}$. Thus, only the assembly of the condensed force column $\mathbf{f}^{r}$ and of the condensed stiffness matrix $\mathbf{K}^{r}$ change due to summation. The force column and stiffness matrix are now expressed as follows:

$$
\begin{aligned}
& \mathbf{f}^{r, s}\left(\mathbf{u}_{r}\right)=-\sum_{i \in S} w_{i} \Psi^{T} \mathbf{f}_{e x t, i}+\sum_{i \in S} w_{i} \Psi^{T} \mathbf{f}_{i}\left(\Psi \mathbf{u}_{r}\right) \\
& \mathbf{K}^{r, s}\left(\mathbf{u}_{r}\right)=\sum_{i \in S} w_{i} \Psi^{T} \mathbf{K}_{i}\left(\boldsymbol{\Psi} \mathbf{u}_{r}\right) \Psi
\end{aligned}
$$

in which $\mathbf{f}^{r, s}$ and $\mathbf{K}^{r, s}$ represent the sampled, condensed force column and the sampled, condensed stiffness matrix respectively. We emphasize that the above way of introducing the summation guarantees that the forces and stiffness (given by Eq. (18) and (19)) are consistent with the approximated (by summation) energy in Eq. (17). The inconsistency introduced e.g. in the force-based approach of Knap and Ortiz (2001) is thus avoided - see Eidel and Stukowski (2009) for a more detailed discussion.

To ensure that the computational cost is substantially reduced, the number of sampling atoms must be much smaller than the total number of atoms $(s \ll n)$. The question arises now which atoms are appropriate sampling atoms, i.e. which atoms need to belong to subset $S$, to ensure that the estimate of the total potential energy is accurate for a given interpolation.

\section{Existing summation rules}

Two general approaches for the selection of sampling atoms are proposed in the QC literature, in which also the computation of the site-energies and weight factors is different. We refer to a specific selection procedure combined with its own computation of site-energies and weight factors as a summation rule. 


\subsection{The local-nonlocal summation rule}

The first summation rule is the local-nonlocal summation rule (Tadmor et al., 1996a,b; Shenoy et al., 1999; Shimokawa et al., 2004). It computes the site-energy of sampling atoms in the fully resolved domain nonlocally, so that all neighboring atoms influence the site-energy of a sampling atom and the exact atomistic model is recovered. This ensures a high accuracy in the fully resolved regions. In the coarse domain however, the site-energy of one sampling atom per triangle is locally computed using the CauchyBorn rule, ensuring a high efficiency. This means that the site-energy is computed as if the sampling atom were located in an infinite crystal which is deformed uniformly. This is accurate only if the deformation fluctuates slowly at the scale of a triangle. Therefore, the site-energy only depends on the repatoms associated with the triangles surrounding that particular sampling atom. An illustration of this summation rule is shown on the left in Fig. 1. The local-nonlocal summation rule leads to the use of a small number of sampling atoms. In the fully resolved regions only the repatoms are selected as sampling atoms and one sampling atom is in principle selected per triangle in the coarse domain (of which the energy is related to the repatoms of the considered triangle (Shenoy et al., 1999)).

However, the coupling of the coarse local region to the fully resolved nonlocal region an internal interface, since not all local sampling atoms are influenced by nonlocal sampling atoms, whereas vice versa this is the case. An example is shown for the local sampling atom indicated in black on the left in Fig. 1. Considering the corresponding cut-off radius, three nonlocal and four local sampling atoms are present within the cut-off region that do not influence the site-energy of this sampling atom. To ensure that this locally computed site-energy is accurate, the fully resolved regions must be relatively large.

The so-called ghost-forces (Shenoy et al., 1999; Miller and Tadmor, 2002), that arise at the internal interface due to the presence of local and nonlocal sampling atoms, can be corrected using corrective procedures, as e.g. proposed by Shenoy et al. (1999); Shimokawa et al. (2004). These corrective procedures allow for a reduction of the fully resolved regions, ensuring an increase of the computational gain. They however need to be updated if

the fully resolved regions evolve, resulting in a increase of complexity and a decrease of the computational gain. 

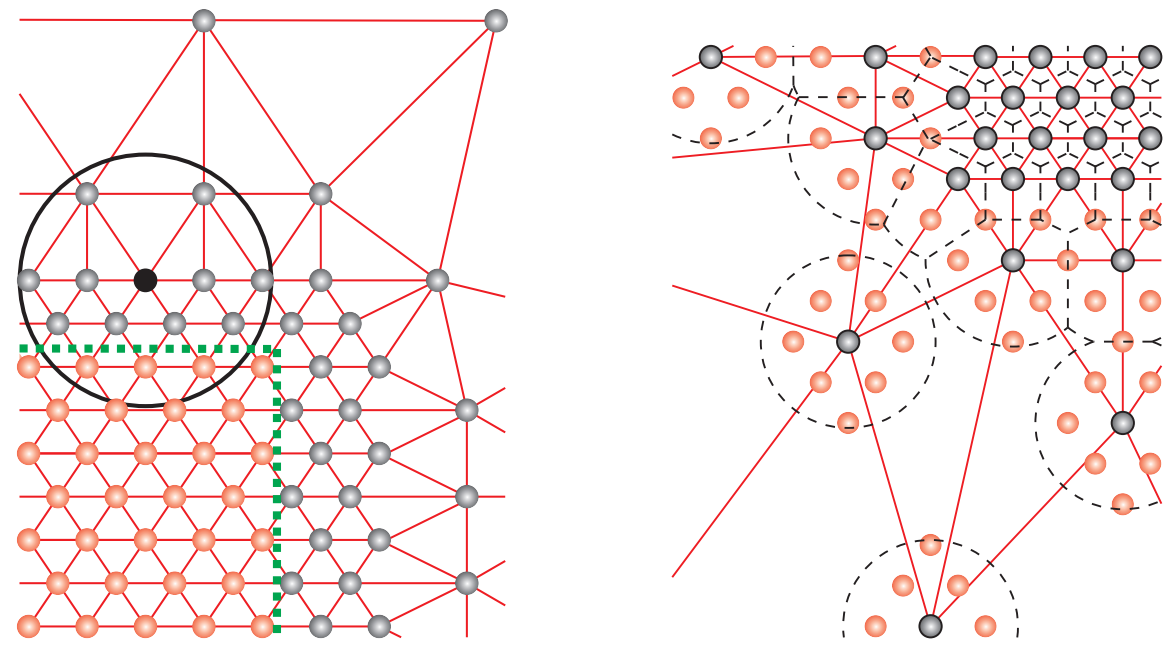

Figure 1: Schematic representation of a two-dimensional atomistic lattice with a triangulation in combination with (left) the local-nonlocal summation rule and (right) the cluster summation rule. (Left) The dark repatoms are part of the coarse domain and the red (light) atoms are repatoms as well as sampling atoms in the fully resolved domain. The cut-off radius around the bold atom is shown. The dashed line represents the location of the internal interface between the fully resolved region and the coarse region. (Right) the dark atoms are repatoms $(R)$ while both dark atoms as well as the other indicated atoms form the sampling atoms $(S)$. The dashed circles mark the clusters of sampling atoms (and are not necessarily related to the cut-off radius).

\subsection{The cluster summation rule}

The second summation rule is the cluster variant, which was introduced by Knap and Ortiz (2001) in terms of forces and reformulated by Eidel and Stukowski (2009) in terms of energy (as is the summation rule proposed in this study). This summation rule computes the site-energy of sampling atoms nonlocally in the fully resolved domains as well as in the coarse domains. Therefore, no internal interface occurs and a seamless transition is obtained towards fully resolved regions.

According to this summation rule, clusters of sampling atoms are selected which are centered at the repatoms $(R \subseteq S \subseteq N)$. The repatoms therefore also form sampling atoms (see the right illustration in Fig. 1). If the clusters tend to overlap, they are truncated so that sampling atoms are only used once. The truncation of the clusters and the nonlocal computation of the sampling atoms' energies ensures that a smooth transition is achieved towards 
the fully resolved regions in which the full atomistic lattice is recovered as shown on the right in Fig. 1. A higher accuracy is achieved, at the expense of computational efficiency, if a larger cluster radius is used. Note that if the cluster radius is set to zero, the repatoms are the only sampling atoms $(S=R)$ and the node-based summation rule of Knap and Ortiz (2001), for which zero-energy modes may occur, is retrieved.

Disadvantages of the cluster summation rule are a relatively low accuracy and large look-up tables. The poor accuracy is caused by the use of one summation weight per cluster $\left(w_{i}\right.$ in Eq. (18) and (19)), as shown by Luskin and Ortner (2008), but also by the mere selection of clusters as is shown below.

Large look-up tables result from the nonlocal site-energy computation of all sampling atoms and increase the bookkeeping costs. On the other hand, the fact that no corrective interface procedures have to be used can be considered as an advantage. The reason is that if adaptivity is included to allow for evolving fully resolved regions, no corrective procedures have to be updated.

\section{Relation between the interpolation and the total potential en- ergy}

In order to obtain an improved summation rule that estimates the total potential energy well without introducing an internal interface, we consider more carefully how the total potential energy of the interpolated system depends on the interpolation. For this purpose, consider a semi-QC method in which interpolation is applied to an atomistic model, but no summation rule is used, i.e. $R \subseteq N$ and $S=N$. All $n$ atoms of the lattice must thus be visited in this semi-QC method to determine the total potential energy, which is exact, albeit for the interpolated lattice. An illustration of the semiQC method is shown in Fig. 2 for a two-dimensional closely packed atomistic lattice (i.e. the distances between every atom and its six nearest neighboring atoms equal one lattice spacing).

Now, consider atom $p$ in Fig. 2. The site-energy of atom $p$ depends on its own displacement and the displacements of its 18 neighboring atoms (encircled in Fig. 2) within its cut-off radius according to Eq. (2). Since all neighboring atoms of atom $p$ are located within or exactly on the edge of the triangle in which atom $p$ is located and the displacements are interpolated 


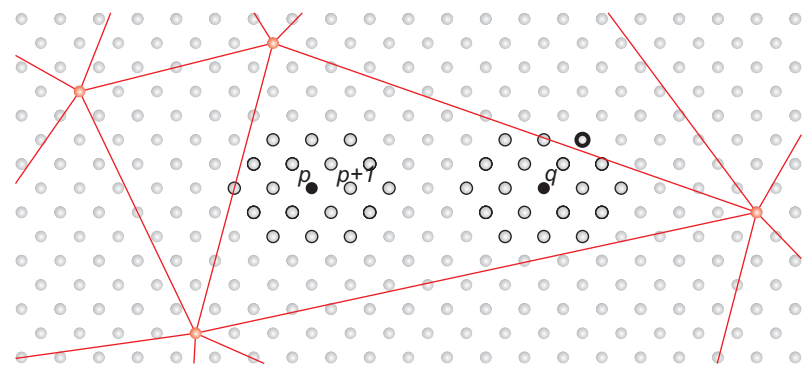

Figure 2: Schematic representation of the semi-QC method: a two-dimensional atomistic lattice with a superimposed triangulation between the repatoms (shown in red). The neighboring atoms of the dark atoms $p$ and $q$ are encircled. The neighboring atom of atom $q$ indicated with the bold circle is located in another triangle than atom $q$.

linearly within this triangle, the site-energy of atom $p$ ultimately depends on the displacements of the three repatoms of the triangle only.

A second important aspect is that because linear interpolation functions are used, the relative displacements of all identical bonds within a particular triangle are equal. This entails that the site-energies of atom $p$ and atom $p+1$ in Fig. 2 are identical, because all their neighboring atoms are located inside the same triangle. As a consequence, the contribution made by atom $p+1$ to the energy of the system is identical to that of atom $p$. This also implies that in Eq. (15) and (16) we have $\boldsymbol{\Psi}^{T} \mathbf{f}_{p}=\boldsymbol{\Psi}^{T} \mathbf{f}_{p+1}$ and $\boldsymbol{\Psi}^{T} \mathbf{K}_{p} \boldsymbol{\Psi}=\boldsymbol{\Psi}^{T} \mathbf{K}_{p+1} \boldsymbol{\Psi}$, respectively.

The neighboring atoms of atom $q$ in Fig. 2, however, are not all located within the same triangle. Thus, the site-energy of atom $q$ depends not only on the repatoms of the triangle in which atom $q$ is located, but also on the repatoms of a neighboring triangle. This means that the potential site-energy of atom $q$ can be different from those of atoms $p$ and $p+1$ and thus also $\boldsymbol{\Psi}^{T} \mathbf{f}_{p} \neq \boldsymbol{\Psi}^{T} \mathbf{f}_{q}$ and $\boldsymbol{\Psi}^{T} \mathbf{K}_{p} \boldsymbol{\Psi} \neq \boldsymbol{\Psi}^{T} \mathbf{K}_{q} \boldsymbol{\Psi}$.

The potential energy of the triangle in which atoms $p, p+1$ and $q$ are located remains thus identical if, instead of computing the site-energies of all atoms, the site-energy of atom $p$ is computed and multiplied by the number of atoms that have their neighbors in the triangle ( $w_{i}$ in Eq. (18) and (19)). In principle, the sampling atom may be chosen arbitrarily among those that have their neighbors within the triangle. We refer to such a sampling atom as a central sampling atom. The site-energies of the atoms that have one or more neighboring atoms in a different triangle, such as atom $q$, must still be 
computed individually if the potential energy is to be exact. Such sampling atoms which only sample their own site-energy (i.e. $w_{i}=1$ ) are referred to as discrete sampling atoms.

For small triangles in which all atoms have one or more neighbors in different triangles all atoms are discrete sampling atoms (see Fig. 3). Furthermore, in the triangles in fully resolved regions this type of summation leads to the use of only the repatoms as discrete sampling atoms, so that the atomistic model is automatically recovered (see Fig. 3). In large triangles, many atoms may be represented by the central sampling atom and the corresponding weight factor $w_{i}$ may thus be large. Along the edges (faces) of the triangles (or tetrahedra) a band of discrete sampling atoms however remains.

The focus of the exact "summation" is thus on the inner part of the triangles. The repatoms, or the atoms around the repatoms, which have many neighbor interactions crossing triangle edges, are thus not appropriate to sample the site-energies of the inner atoms which have all their neighbor interactions within the same triangle. However, in the cluster summation rule exactly these atoms with many interactions across triangle edges are used for sampling. This means that not only the specific computation of the weight factors in the cluster summation rule leads to inaccurate results, as shown by Luskin and Ortner (2008), but the mere selection of clusters of sampling atoms around the repatoms itself is questionable.

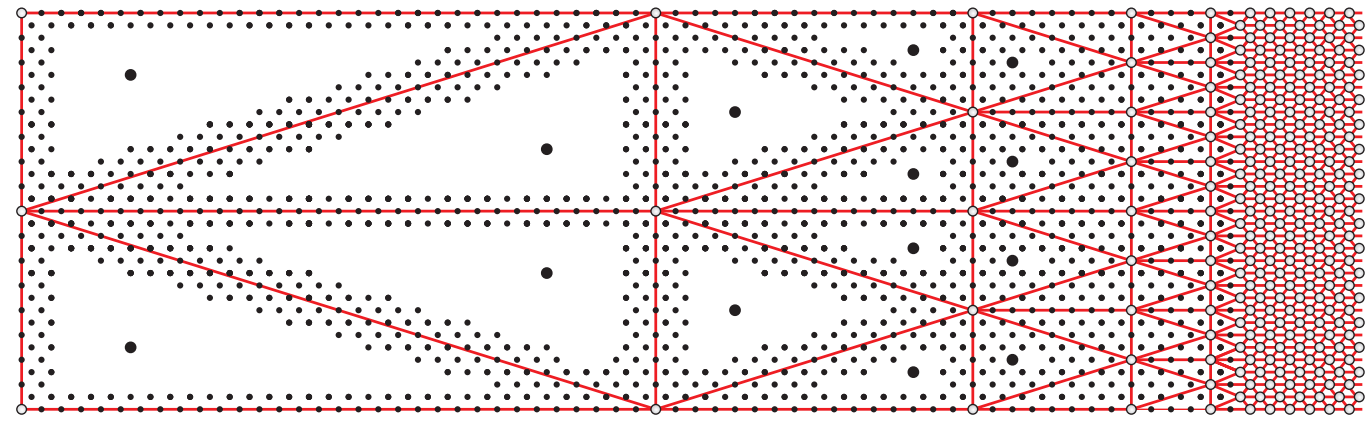

Figure 3: A summation rule that recovers the exact potential energy applied to a twodimensional closely packed atomistic lattice including a fully resolved region on the right. The open circles represent the repatoms as well as discrete sampling atoms, the large dark circles represent central sampling atoms and the small dark circles indicate discrete sampling atoms. 
As can be seen in Fig. 3, the exact summation leads to the use of a large number of sampling atoms due to the bands of discrete sampling atoms at the edges of the triangles. For lattice models that only contain nearest neighbor interactions, such as two-dimensional structural lattice models, such a summation rule is computationally viable, as shown by Beex et al. (2011), because these bands are thin. For atomistic models in which not only nearest neighbor interactions are present, this type of summation would result in rather large (and hence expensive) bands of discrete sampling atoms. Furthermore, if three-dimensional crystals are considered, zones of thickness

$2 r_{\text {cut }}$ are needed along all faces of the interpolation tetrahedra, leading to an even larger number of discrete sampling atoms.

\section{Central summation rule}

The summation rule explained above is exact. For atomistic crystals it is however too inefficient because it leads to many sampling atoms, i.e. $s \approx n$. The central summation rule proposed in this section aims to be more efficient so that $s \ll n$. For this reason, only one internal sampling atom is in principle selected in each triangle. Although this introduces a certain degree of approximation, the central summation rule is based on the understanding of how the total potential energy relates to the interpolation and its accuracy is therefore still high compared to a number of existing summation rules.

\subsection{Selection of sampling atoms}

We take as a starting point the exact summation as discussed above. Rather than keeping track of all interatomic interactions that cross triangle edges (see Fig. 4) by defining discrete sampling atoms along these edges, these atoms are now also represented by the respective central sampling atoms. In this way the number of sampling atoms is largely reduced but the total potential energy is estimated, instead of exactly determined. The approximation introduced is acceptable if the difference in deformation between neighboring triangles is small. Where large differences occur, a larger error is made, or the triangulation should be refined.

In the central summation rule the atom closest to the incenter of the triangle (or tetrahedron) is selected as the central sampling atom of each interpolation triangle (i.e. belongs to $S$ ). The reason that the incenter of the triangles is used is that it marks the location of the point that is located 


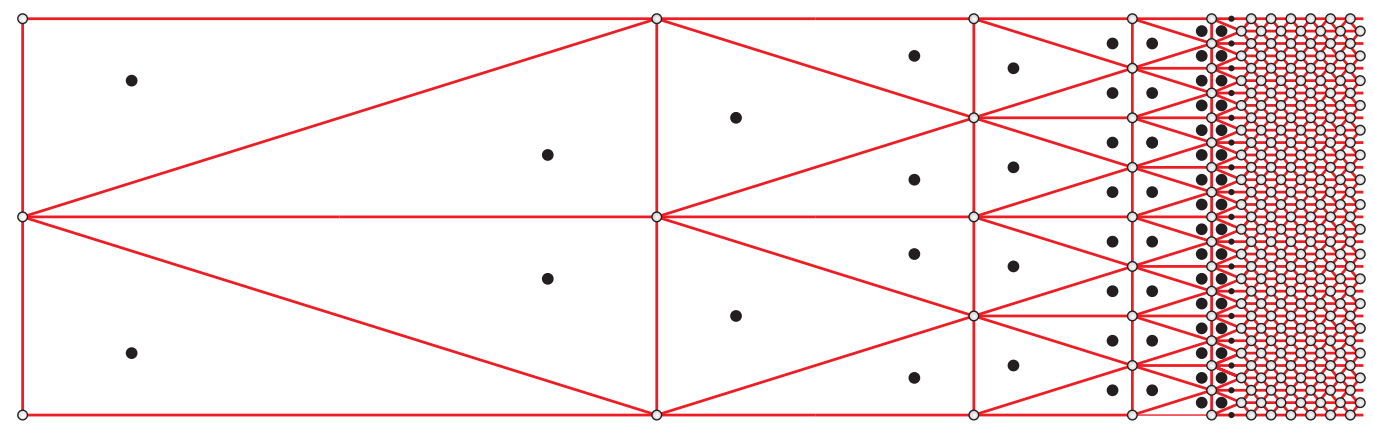

Figure 4: Illustration of the proposed central summation rule for a two-dimensional closely packed atomistic lattice including a fully resolved region on the right. The open circles represent the repatoms as well as discrete sampling atoms, the large dark circles represent central sampling atoms and the small dark circles indicate discrete sampling atoms.

furthest from each triangle edges (see Fig. 5). It therefore marks the location that has the least probability of neighbors in other triangles.

In case a significant number of neighbors of the central atom are present in adjacent triangles, the central atom is the one that has its neighbors most evenly spread over the adjacent triangles. If another atom is used for sampling (in case only one sampling atom is to be selected for computationally efficiency), that is closely located to one adjacent triangle but further away from the other adjacent triangles, it may have a disproportional number of neighbors in the closest adjacent triangle, and no or few neighboring atoms in the other adjacent triangles. For symmetric triangulations this selection ensures a symmetric selection of sampling atoms.

In case a triangle or tetrehadron only contains atoms on its corner nodes, edges and faces, and no atoms within the triangle or tetrahedron, all atoms are selected as discrete sampling atoms $\left(w_{i}=1\right)$. This means thus that all central sampling atoms (with $w_{i} \geq 1$ ) reside inside the triangle. This can be seen for the band of triangles in the coarse domain next to the fully resolved domain in Fig. 4. Sampling atoms on top of an edge or face may not be selected as a central sampling atom, since this would induce alternating patterns of weight factors for the central sampling atoms that can lead to saddle-point solutions and unstable equilibria. 


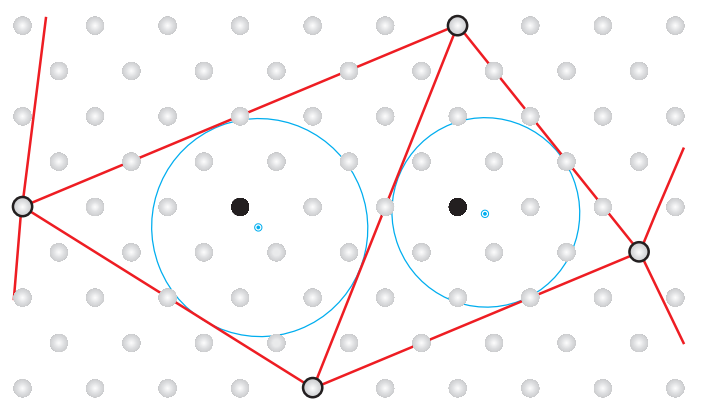

Figure 5: Schematic representation of the central sampling atom selection (large, dark dots) which are located closest to the incenter (small, blue dots) of two triangles which are marked by the two blue circles in the triangles. Repatoms (encircled) act as discrete sampling atoms and the remaining atoms (light grey) are accounted for in the weight factors of the corresponding central sampling atoms.

\subsection{Weight factors}

As indicated before, the discrete sampling atoms only represent themselves and therefore have a weight factor $w_{i}=1$. The remaining atoms in a particular triangle are summed in the weight factor of the central sampling atom of that triangle, including the central sampling atom itself. Atoms located on a triangle edge are accounted for by $50 \%$ each in the weight factors of the central sampling atoms of the two triangles.

For three-dimensional atomistic crystals, the contributions of the atoms on a tetrahedral face are equally divided between the central sampling atoms of the adjacent tetrahedra. For atoms located on tetrahedron edges the weight factors are determined by the angle of the two faces intersecting the edge.

\subsection{Site-energy computation}

The computation of the sampling atoms' site-energies is formulated in an energy setting according to Eq. (6) and Eq. (10), i.e. no force-based formulation is used. All site-energies are furthermore computed nonlocally to ensure that the interatomic bonds which cross triangle edges are correctly incorporated. This means that all neighboring atoms of all (central and discrete) sampling atoms must be addressed to ensure that the site-energies of the sampling atoms are correctly computed. For central sampling atoms in large triangles a nonlocal computation of the site-energy equals the local computation using the Cauchy-Born rule, since all neighbors are located within the 
same triangle. However, the neighbors of central sampling atoms in small triangles and tetrahedra may be located in other interpolation elements and their nonlocal computation thus results in a dependence on repatoms of several triangles or tetrahedra. Moreover, this is always the case for discretely sampled atoms in particular for repatoms.

An efficient and straightforward algorithm of the central summation rule is guaranteed by ensuring that all triangles are treated in the same fashion (see Table 1). For this reason the repatoms are also used as discrete sampling atoms in large triangles, although they hardly contribute to the sampled energy of large triangles since their weight factors $\left(w_{i}=1\right)$ are small compared to the weight factors of the central sampling atoms. It is possible to avoid selecting the repatoms of large triangles and tetrahedra as discrete sampling atoms, but this introduces an extra user-defined parameter in the summation rule, at comparatively little computational gain. Because the aim here is to obtain a straightforward and unambiguous summation rule, the repatoms of large triangles are used as well as discrete sampling atoms. For small triangles around the fully resolved domains, these discrete sampling atoms are significant and they assure a smooth transition from fully resolved domains to coarse domains.

No spurious surface energies occur at the edges of the model due to the discrete sampling atoms in large triangles since they hardly contribute for large triangles (tetrahedra). Furthermore, spurious surface energies can in most cases be avoided by using appropriate boundary conditions (e.g. periodic boundary conditions) on all repatoms of the outer triangles of the model (see e.g. the benchmark used by Miller and Tadmor (2009) and the numerical example in section 6.1.2).

The advantage of this summation rule is that as a result of the nonlocal computation of all site-energies no internal interface between the interpolated and fully resolved domain exists. A natural and gradual transition is obtained from a near-local QC method in coarsely discretized regions to a fully nonlocal method in fully resolved regions. No interface corrections, for which additional assumptions are often necessary (Shenoy et al., 1999), have to be implemented. Although the nonlocal computation of site-energies requires look-up tables (containing sampling atoms and their neighbors) at the expense of some bookkeeping, the fact that no internal interface corrections have to be updated guarantees efficient analyses in which fully resolved regions may transform or move significantly. 
Table 1: Algorithm for the central summation rule for three-dimensional atomistic crystals.

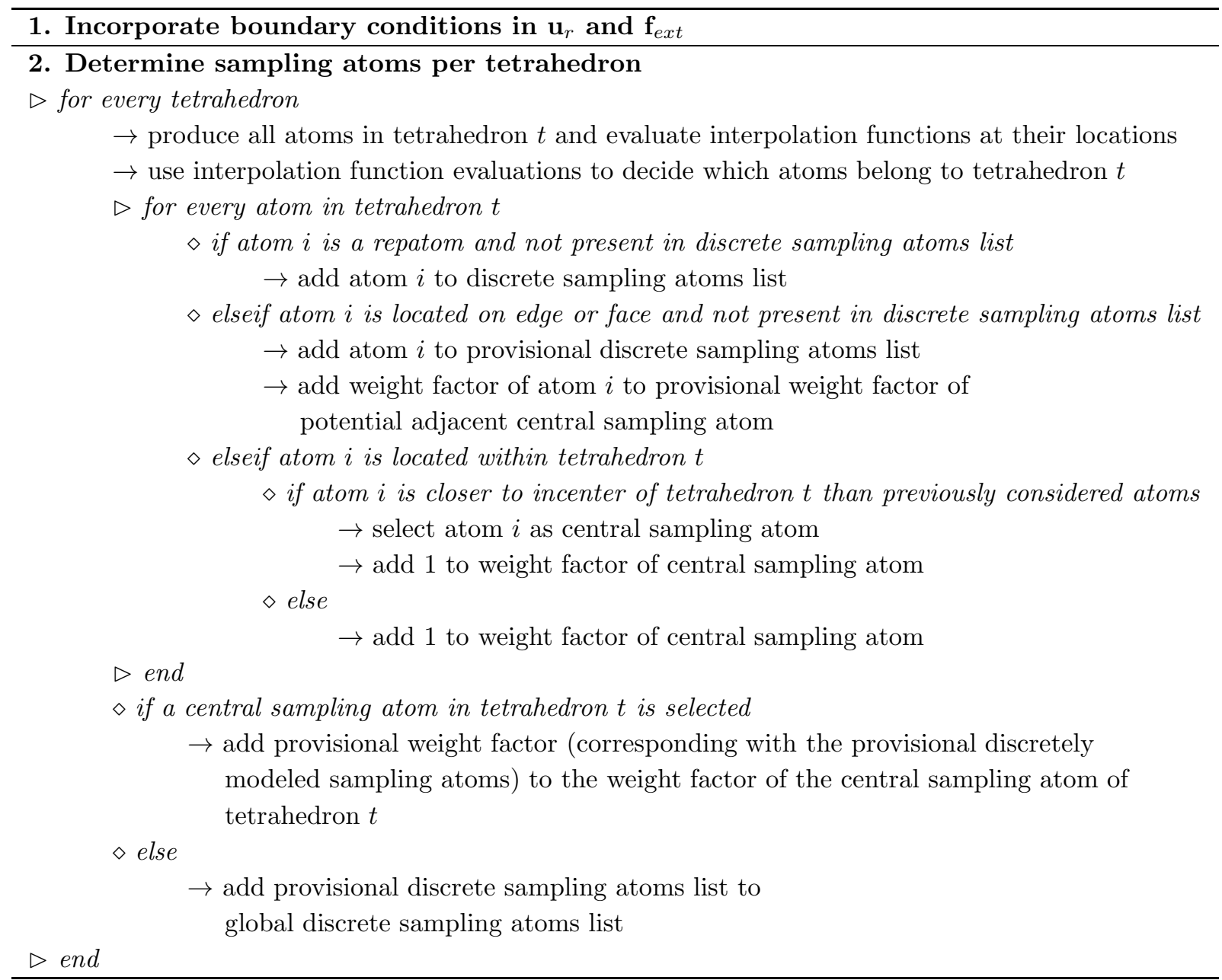

3. Recover missing neighbors of all sampling atoms with corresponding evaluated interpolation functions

4. Assemble condensation matrix $\Psi$ with evaluated interpolation functions of sampling atoms and neighbors

5. Use Newton-Raphson procedure

$\triangleright$ for each increment until residual meets tolerance

- Produce condensed, summed force column $\mathbf{f}^{r, s}$ and stiffness matrix $\mathbf{K}^{r, s}$ according to

Eq. (18) and (19)

- Partition $\mathbf{f}^{r, s}$ and $\mathbf{K}^{r, s}$ and update solution according to Eq. (14) 


\section{Results}

\subsection{Vacancy in a two-dimensional crystal using the Lennard-Jones potential}

As a first numerical example to evaluate the central summation rule, a two-dimensional closely packed atomistic crystal with a vacancy in its center is analyzed. The crystal contains 251 by 285 rows of atoms in horizontal and vertical direction respectively (i.e. a total of 71,392 atoms). The LennardJones (6-12) potential (Lennard-Jones, 1937) is used for the interatomic pair potential, $E_{i j}$ in Eq. (2). This potential has its minimum at the interatomic lattice spacing, $r_{0}$, as depicted in Fig. 6 . The cut-off radius is set to 2.23 lattice spacings, implying that each atom interacts with 18 neighboring atoms.

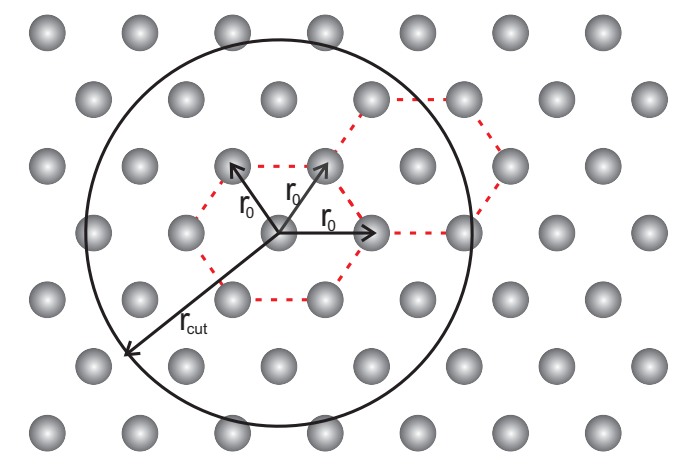

Figure 6: Schematic representation of the two-dimensional closely packed atomistic crystal. The atomistic lattice spacing - at which the interatomic Lennard-Jones potential has its minimum - is given by $r_{0}$. The interaction region of a single atom is shown by the circle with cut-off radius $r_{c u t}$. Two unit cells are depicted by the dashed hexagonals.

An example of a triangulation including a fully resolved region around the vacancy is shown in Fig. 7. The crystal is loaded to a uniaxial strain of $0.5 \%$ in $\left[\begin{array}{ll}1 & 0]\end{array}\right.$ (horizontal) direction. No vertical strain is allowed. Displacement boundary conditions in accordance with this uniaxial strain state are applied to the repatoms of the band of slim triangles that surround the model. This band has a thickness which exceeds twice the cut-off radius to ensure that no edge effects occur (see Fig. 7). In all triangulations considered this band is the same. The size of the fully resolved region around the vacancy is varied using different triangulations. The transition to the coarse domain is made by doubling the triangle size for every extra ring of triangles away from the fully resolved region. 

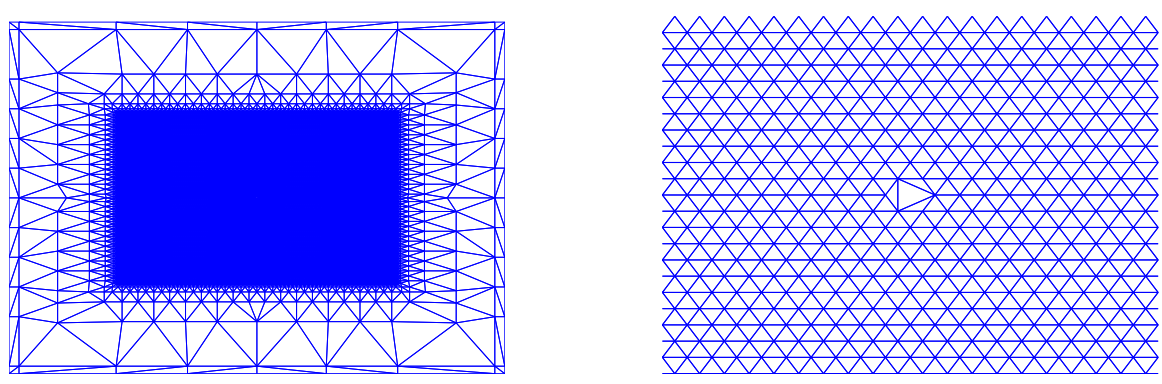

Figure 7: The entire triangulation for the two-dimensional closely packed atomistic crystal with a fully resolved region of $71 \times 70$ unit cells around the vacancy that serves as a reference (left) and a zoom around the vacancy (right). The [1 0$]$ direction corresponds to the horizontal direction and the [0 1$]$ direction is oriented at an angle of $60^{\circ}$ relative to the horizontal direction and thus at an angle of $-30^{\circ}$ relative to the vertical direction.

\subsubsection{Reference solution}

The semi-QC variant as described in Section 5 serves as a reference for the assessment of the central summation rule. All atoms in the lattice are used to determine the total potential energy exactly in this reference case $(S=N)$ and thus, no error due to summation occurs. The only error left resides in the interpolation, for which a large fully resolved region of $71 \times 70$ unit cells in horizontal and vertical direction respectively ensures that this error remains small (see Fig. 7). The predicted relative [1 0 ] (horizontal) displacement components, $u_{x}^{r e l}$, which show the influence of the introduced vacancy, are shown as a function of the location of the repatoms in Fig. 8. This relative displacement is obtained by subtracting the horizontal displacements of the same model without a vacancy (loaded with the same boundary conditions) from the computed displacements:

$$
u_{x}^{r e l}\left(\vec{X}^{i}\right)=u_{x}\left(\vec{X}^{i}\right)-E_{x x} X_{x}^{i}
$$

where $\vec{X}^{i}$ is the position vector indicating the original location of atom $i$, $u_{x}\left(\vec{X}^{i}\right)$ is the horizontal component of the displacement vector of atom $i$ and $E_{x x}$ is the applied overall strain in [ 10$]$ (horizontal) direction. Two distinct peaks, which both have an amplitude of $0.0068 r_{0}$, can be observed at the 
two repatoms located left and right of the vacancy. In $\left[\begin{array}{ll}1 & 0\end{array}\right]$ direction the fluctuation caused by these peaks decays slower than in the vertical direction.
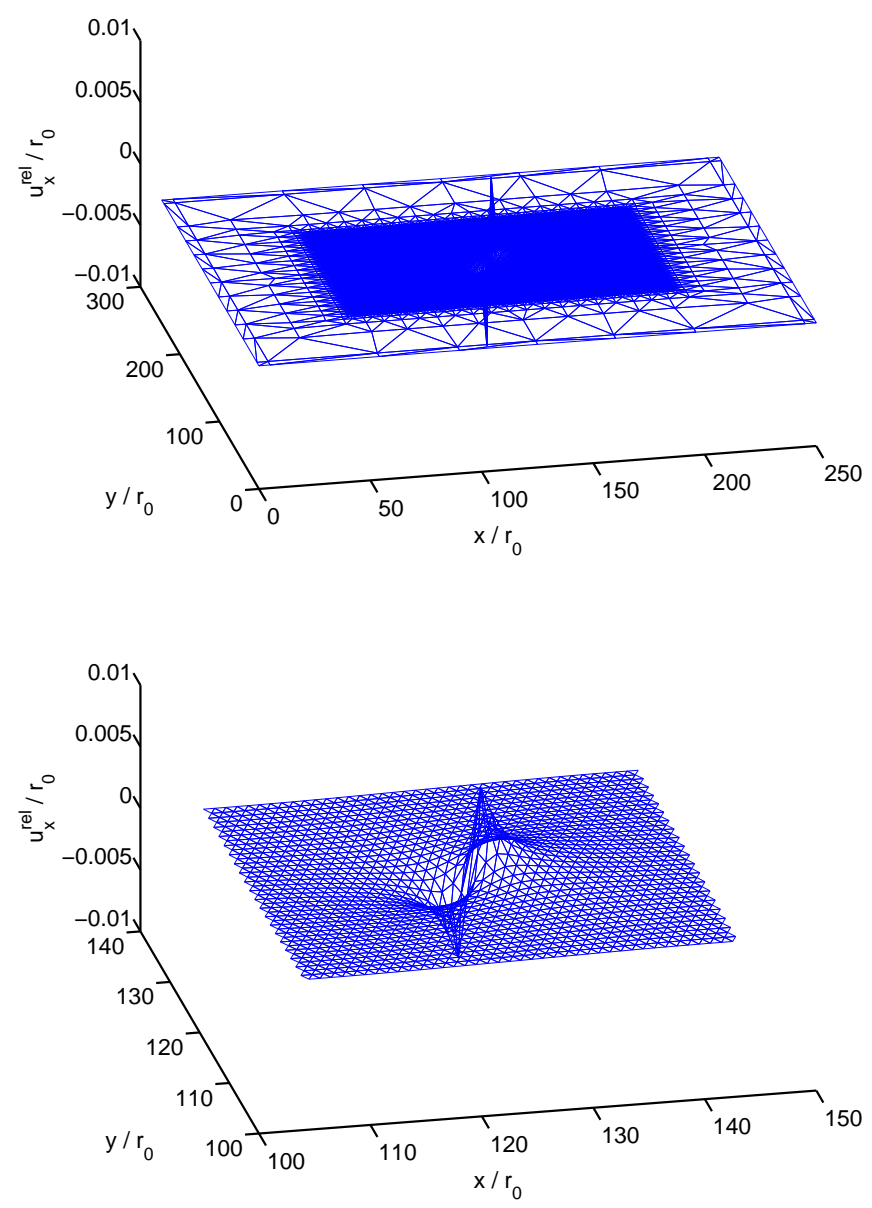

Figure 8: The displacement field in [1 0 ] (horizontal) direction of the semi-QC method with a fully resolved region of $71 \times 70$ unit cells in horizontal and vertical direction. The predicted relative horizontal displacements are shown, normalized by $r_{0}$, in all repatoms (top) and a zoom around the vacancy (bottom).

\subsubsection{Solution predicted with the central summation rule}

We illustrate the effect of summation first on a triangulation with a (very small) fully resolved region of $3 \times 4$ unit cells in horizontal and vertical 
direction respectively. The sampling atoms selected according to the central summation rule are marked in Fig. 9. After the identification of the sampling atoms and the recovery of the missing neighbors of the sampling atoms, the atom at the location of the vacancy was removed (see Fig. 9, right).
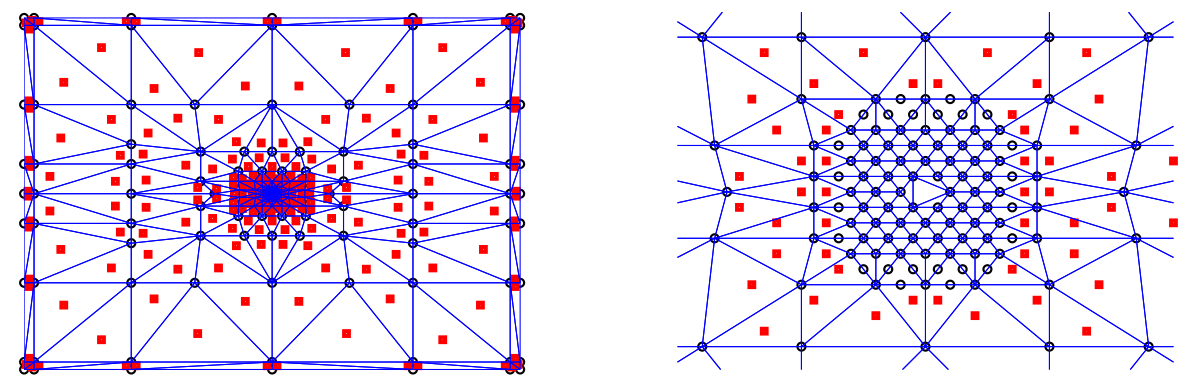

Figure 9: The entire triangulation for the two-dimensional atomistic crystal with a fully resolved region of $3 \times 4$ unit cells including the sampling atoms selected according to the central summation rule (left) and a zoom of the fully resolved region of the fully resolved region (right). Discrete sampling atoms (including repatoms; $w_{i}=1$ ) are presented as black circles and central sampling atoms $\left(w_{i}>1\right)$ as red squares. Neighboring atoms of the sampling atoms are not shown.

The results computed with the central summation rule for the triangulation of Fig. 9 are shown in Fig. 10. The peaks on the left and right side of the vacancy are in reasonable agreement with the reference results of Fig. 8; their amplitude is $0.0081 r_{0}$. This corresponds with a maximum error of the displacement of these two repatoms of $18.6 \%$ for this triangulation. A semiQC simulation for the triangulation of Fig. 9 (not shown here), in which no error due to summation occurs, has indicated that the error due to the interpolation is only $1.0 \%$ for this triangulation. It can thus be concluded that the maximum error of $18.6 \%$ is mainly caused by the summation.

The predicted displacement field however also shows discrepancies with the reference solution in Fig. 8 in and around the fully resolved region. These discrepancies with a maximum amplitude of $0.0053 r_{0}$ are caused by the error due to summation, since they are not present in the results of the semiQC variant applied to this triangulation. The fully resolved region of the triangulation shown in Fig. 9 is apparently so small that in the first ring of triangles in the coarse domain the site-energies of the atoms within one triangle differ substantially from each other. This use of relatively large 

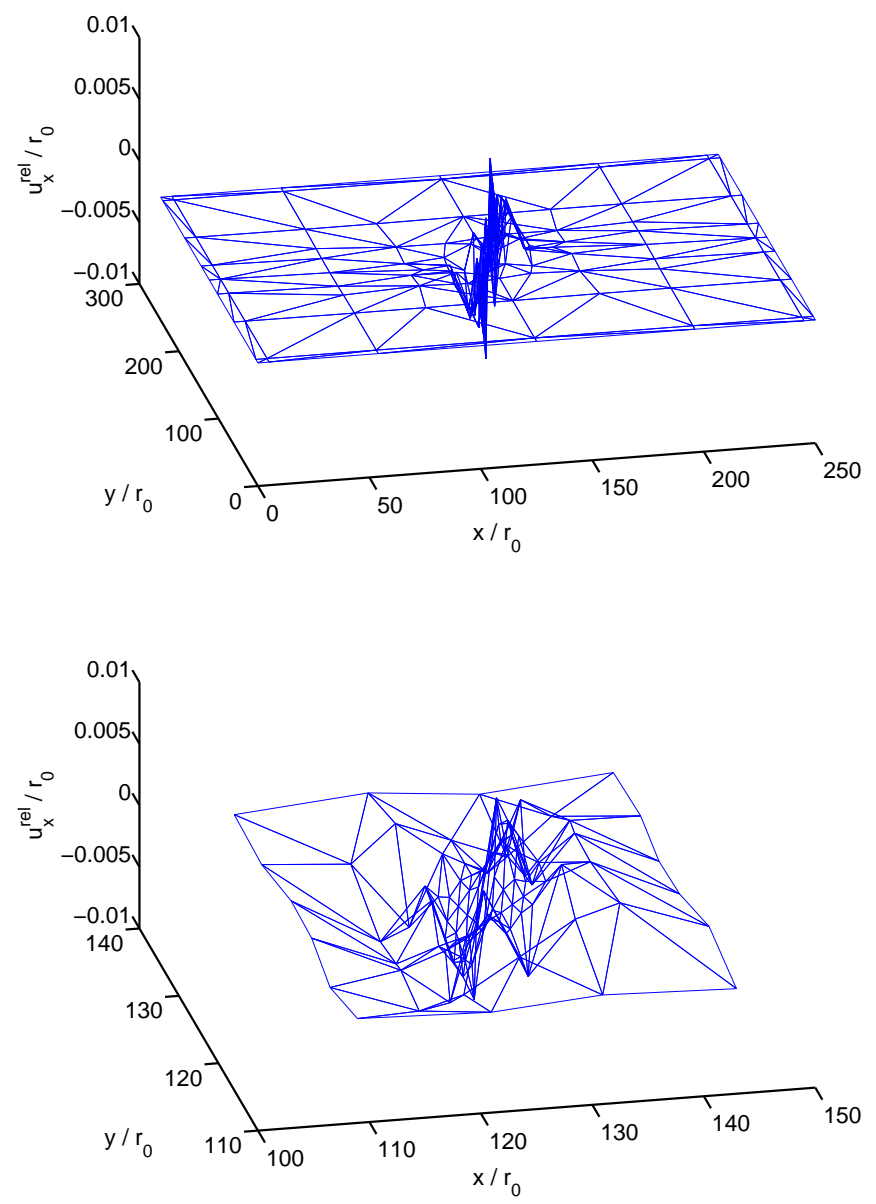

Figure 10: The relative displacement field in [1 0 ] (horizontal) direction computed for the triangulation of Fig. 9 with a fully resolved region of $3 \times 4$ unit cells. The predicted relative horizontal displacements in terms of $r_{0}$ are shown as a function of the repatoms of the entire triangulation (top) and a zoom around the vacancy (bottom).

triangles in regions where atoms behave differently leads to relatively large summation errors, as could be expected. In the coarse domain however, the summation rule captures the uniaxial strain deformation well, as can be seen on the left in Fig. 10.

A straightforward way to improve the predicted displacement field in the region of interest (the region around the vacancy) is to enlarge the fully 
resolved domain. This ensures that the error due to summation only occurs in regions where the site-energies of the atoms vary little, as assumed in the summation rule. On the left in Fig. 11 the maximum errors are presented for triangulations with different sizes of fully resolved regions. The error drops rapidly upon increasing the size of the fully resolved region. If it is sufficiently large, so that the coarse domain, in which the true summation occurs, starts where the site-energies of the atoms are almost identical, the error remains below $5 \%$. This degree of accuracy is reached for fully resolved regions larger than $7 \times 12$ unit cells.
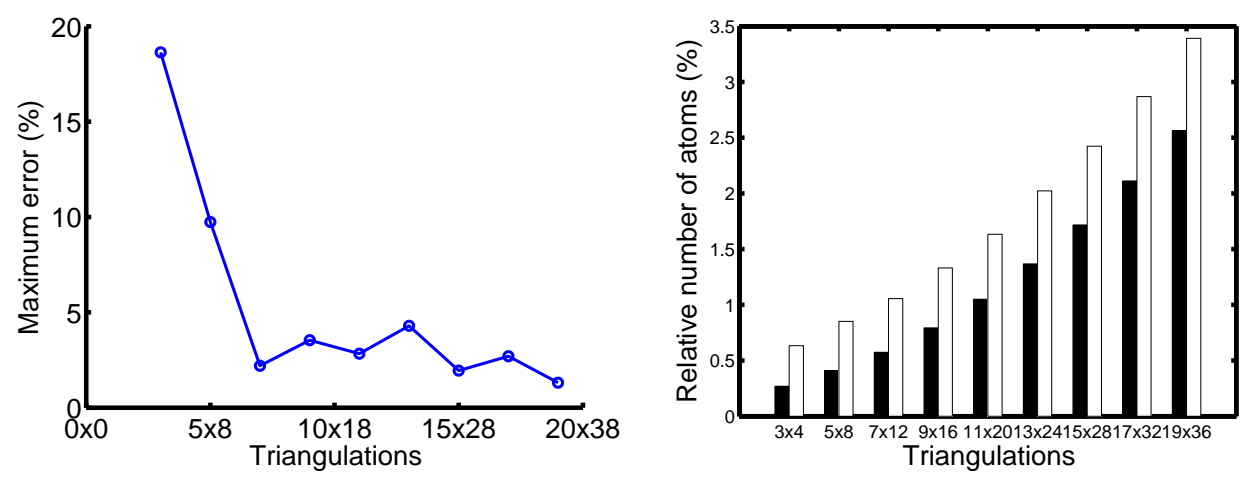

Figure 11: (Left) the maximum error at one of the two repatoms at the peaks of the displacement field in [1 0 ] (horizontal) direction computed using the central summation rule for triangulations with different sizes of fully resolved regions. The maximum error is shown as a function of the sizes of the fully resolved regions. The shapes of the fully resolved regions are kept as square as possible. (Right) the relative number of repatoms (black) and sampling atoms (white) for the triangulations with different sizes of fully resolved regions.

The fact that the maximum error does not decrease to zero for increasing fully resolved regions (Fig. 11) is caused by the non-zero relative displacements that remain in the coarse domain, just outside the fully resolved region (see Fig. 12). The maximum amplitude of these relative displacements for the results shown at the bottom in Fig. 12 is $0.0079 r_{0}$. This is close to the obtained value reached at the maximum peak next to the vacancy. The reason for these discrepancies is that a number of neighboring atoms of the central sampling atoms are located in different triangles for these small triangles around the fully resolved region (see Fig. 9). If the fully resolved region is not too small, however, this error at the edge of the fully resolved region has 

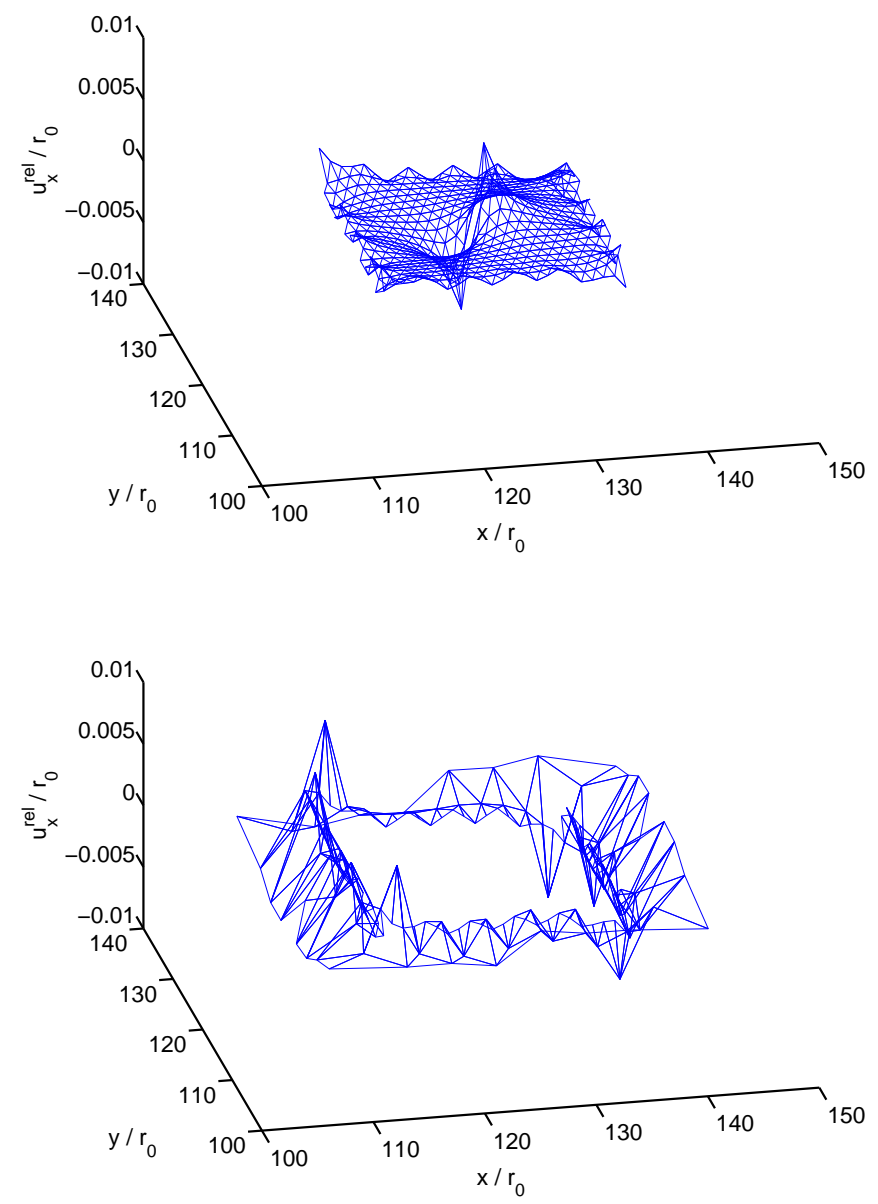

Figure 12: The displacement field in [1 0 ] (horizontal) direction of the central summation rule for the triangulation with a fully resolved region of 11 by 20 unit cells, split up into the fully resolved region (top) and the first two rings of triangles in the coarse domain around the fully resolved regions (bottom). The predicted relative horizontal displacements, normalized by $r_{0}$, are shown as a function of the position of the repatoms.

little influence on the solution in the central region of interest (in this case the solution around the vacancy).

As can be seen in the diagram on the right in Fig. 11, the computational efficiency of the summation rule, expressed in terms of the number of repatoms and sampling atoms relative to the number of total atoms present 
in the crystal, is high and remains high for increasing fully resolved regions. The computational efficiency of the summation rule will be larger if the ratio between the coarse domain (in which many atoms are summed) and the fully resolved domain is larger. Such a trend is shown for the summation rule that recovers the exact potential energy for structural lattices with nearest neighbor interactions by Beex et al. (2011).

\subsubsection{Solution predicted with the cluster summation rule}

It is interesting to compare the results of the proposed central summation rule to those computed with the cluster summation rule. The cluster summation rule, like the central summation rule proposed here, allows a seamless coupling between coarse domains and fully resolved domains.

The cluster summation rule that is used here is the improved one as proposed by Eidel and Stukowski (2009), which is formulated in terms of energy (like the summation rule presented here). The hybrid correction strategy proposed by Eidel and Stukowski (2009) is not used. The reason is that all atoms have to be considered for this and the purpose of QC methodologies is not to use all atoms because that may well be computationally prohibitive.

The cluster summation rule is applied to the triangulation with a fully resolved region of 11 by 20 unit cells. The boundary conditions are identical to those in the previous simulations. The cluster radius is set to 3 atomistic lattice spacings $\left(3 r_{0}\right)$, which means that a cluster contains 37 sampling atoms if it is not truncated because of overlap with another cluster. This leads to the relative use of $6.8 \%$ sampling atoms while the central summation rule only needs $1.7 \%$ sampling atoms for this triangulation (see right diagram in Fig. 11).

The displacement field computed with the cluster summation rule is presented in Fig. 13. It corresponds for the greater part with the displacement field obtained by Eidel and Stukowski (2009) for the two-dimensional cluster computation without the hybrid correction strategy. The correction strategy involves a computation of the interpolated lattice in which all atoms must be incorporated and thus no summation is used (as in the aforementioned semi-QC method). Since summation rules in the QC method are aimed at avoiding computations in which all atoms are incorporated and since no correction approach is required for the central summation rule, the correction strategy is not used here for the comparison between the cluster summation rule and the central summation rule.

The cluster summation rule without the hybrid correction strategy is 

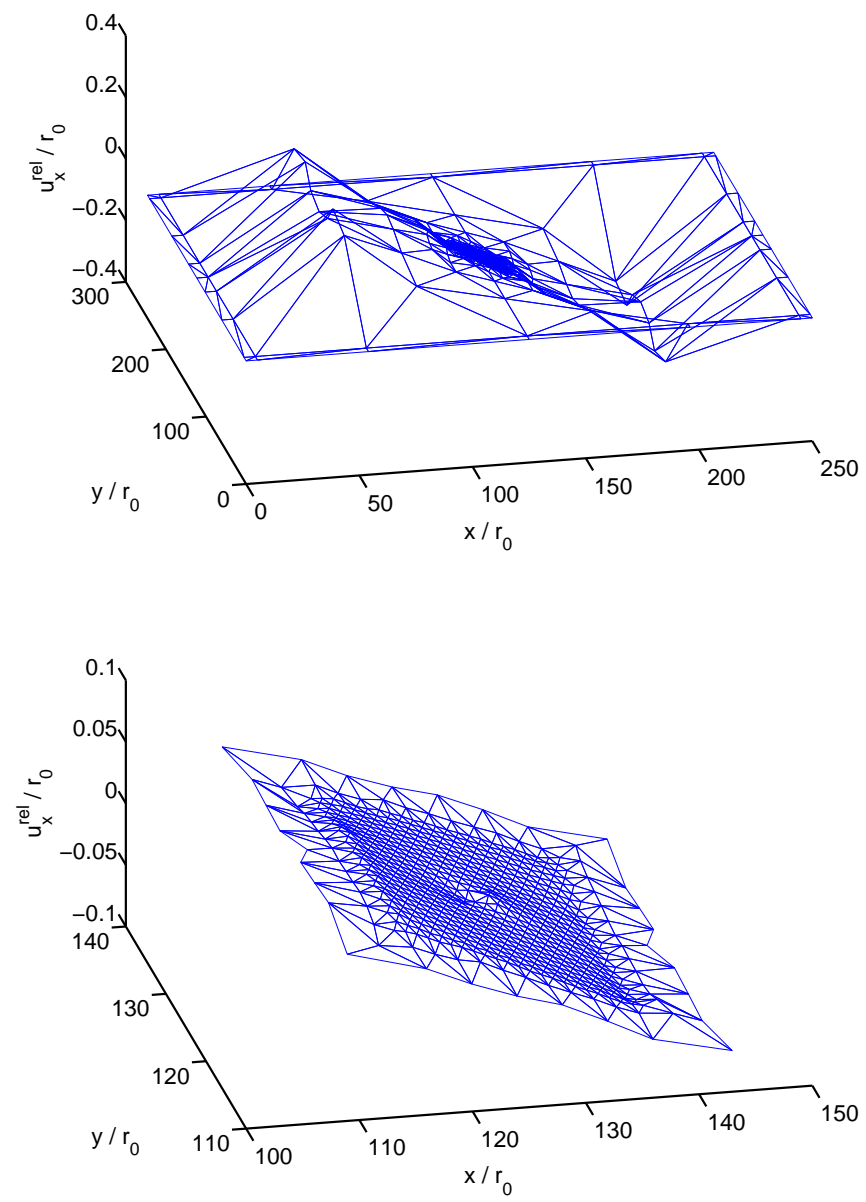

Figure 13: The relative displacement field in [1 0$]$ (horizontal) direction computed with the cluster summation rule for the triangulation with a fully resolved region of 11 by 20 unit cells. The predicted relative horizontal displacements scaled by $r_{0}$ are shown as a function of the repatoms of the entire triangulation (top) and a zoom around the vacancy (bottom). Note the different vertical scales compared with Fig. 8, 10 and 12.

clearly not able to accurately capture the uniform strain applied to the crystal. This is not only caused by a poor computation of the weight factors (Luskin and Ortner, 2008), but also by selecting the sampling atoms around the repatoms (as mentioned before). This clearly leads to significant discrepancies in large triangles in which many atoms are present that have 
all their neighbors within the same triangle, while they are sampled by atoms that have many neighbors in other triangles. Although the cluster summation rule computes the sampling atoms' site-energies in the fully resolved region correctly, the error in the coarse domain has such an influence on the displacements of the entire crystal that the relative [ 10$]$ displacements of two peaks in the fully resolved regions equal $-0.0017 r_{0}$ and $0.0007 r_{0}$. This corresponds to a maximum error of $89.7 \%$.

\subsection{Lomer dipole in a two-and-a-half-dimensional crystal using the embedded- atom-method}

The central summation rule is evaluated in this section for a test case in which a Lomer dipole is included in an FCC crystal for Al. This test case is strongly inspired by the benchmark used by Miller and Tadmor (2009), but focuses on a smaller domain to decrease computational time and includes a rectangular fully resolved region to allow for more interaction between the coarse domain and the fully resolved domain. Instead of the LennardJones potential, the Embedded-Atom-Method (EAM) is used for the interatomic interactions, which introduces a higher degree of nonlocality than the Lennard-Jones potential adopted in the previous example. The embedding functional of Mitev et al. (2006) is used here, which ensures that the potential and its derivatives are smooth. For the exact embedding parameters, we refer to Mitev et al. (2006), since they are used without any adjustment.

The crystal lattice considered is approximately $166.9 \AA$ by $165.6 \AA$ in the $\left[\begin{array}{lll}1 & 1 & 0\end{array}\right]$ direction and $\left[\begin{array}{lll}0 & 0 & 1\end{array}\right]$ direction, respectively. The lattice including the triangulation used is shown in Fig. 14. In total 4,658 atoms are considered in the direct lattice simulation, since the lattice spacing $\left(r_{0}\right)$ is $4.14 \AA$. Several planes of atoms are present below and above the depicted plane. The atoms are allowed to displace in a column-wise fashion, with the axes of the columns oriented in the $\left[\begin{array}{lll}1 & -1 & 0\end{array}\right]$ direction.

Two Lomer dislocations are introduced in the center of the domain, at a distance of approximately $23.4 \AA$ from each other in $\left[\begin{array}{lll}1 & 1 & 0\end{array}\right]$ direction (see Fig. 14). The dipole configuration is created using isotropic elastic displacement fields (Hirth and Lothe, 1992) as initial guess and subsequently performing equilibrium iterations. To ensure that no edge effects occur, boundary layers of a width of $12.4 \AA$ at the four sides of the model are constrained in both the direct lattice computation and the quasicontinuum computation (see Fig. 14). The width of these layers is larger than $2 r_{c u t}$, since $r_{c u t} \approx 6.03 \AA$. The isotropic elastic displacement fields are not applied to the boundaries. 
Consequently, the dislocations are not stress-free. This is however irrelevant for the comparison between the direct lattice computation and the $\mathrm{QC}$ computation including the central summation rule.

In contrast to the previous and the next numerical example in which the Newton-Raphson procedure is used to minimize the potential energy, both the direct lattice computation and the $\mathrm{QC}$ computation in this test case are solved using the conjugate gradient method, because the Newton-Raphson procedure did not converge appropriately for this test case. A fixed step size of $10^{-6}$ is used.
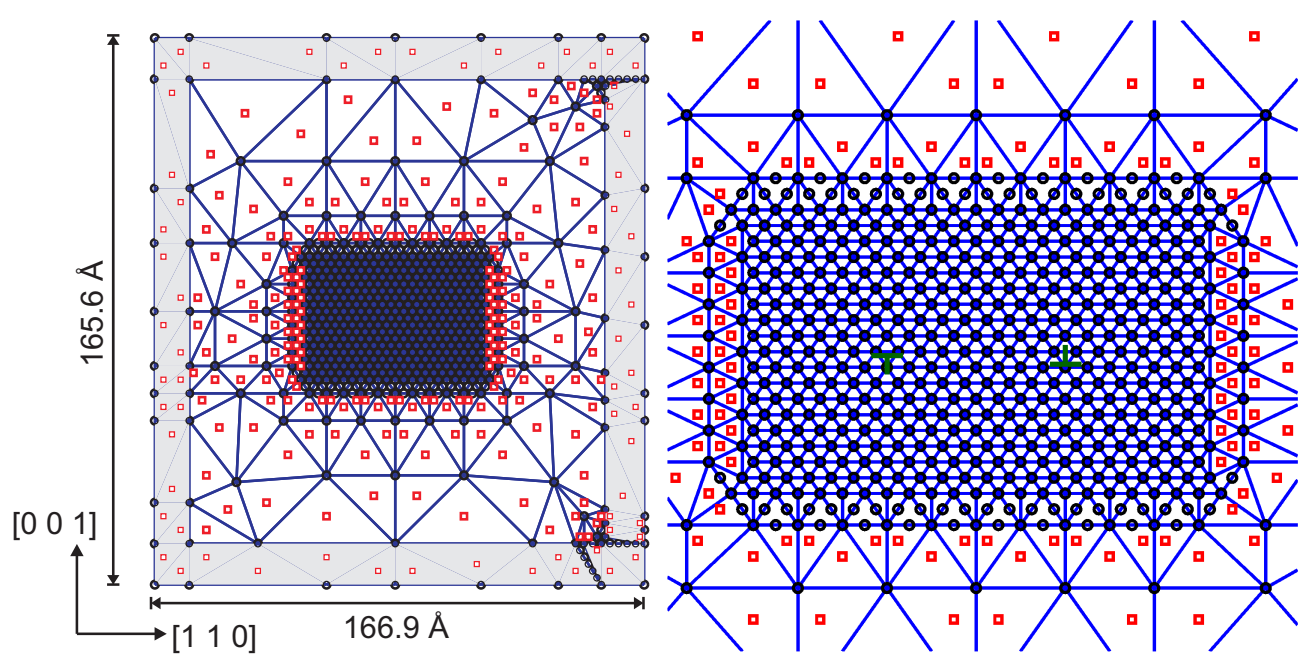

Figure 14: The triangulation used to model the Lomer dipole including the internal sampling atoms (squares) and discrete sampling atoms (circles). Left: the entire triangulation including boundaries of width $12.4 \AA$ (grey) to ensure that no edge effects occur. Right: zoom around the fully resolved region including the locations of the two Lomer dislocations. Note that not all shown (sampling) atoms are located in the same plane.

\subsubsection{Reference solution}

The reference solution used to evaluate the quality of the central summation for this example is the direct lattice computation. The solution predicted with the direct lattice computation is shown on the left in Fig. 15. The Lomer dipole is not stress free which can be observed by the non-zero vertical ( $\left.\left[\begin{array}{lll}1 & 0 & 0\end{array}\right]\right)$ displacement components in the vicinity of the bands surrounding the model. This is in contrast to the reference solution of Miller and Tadmor (2009) and 
is a result of the significantly smaller model size. Another difference with the reference results of Miller and Tadmor (2009) is that the maximum and minimum vertical displacement components are smaller $(-0.63 \AA$ instead of $-0.71 \AA$ and $0.61 \AA$ instead of $0.71 \AA$ ), which is caused by the smaller model size and the smaller distance between the two dislocations.

\subsubsection{Solution predicted using the central summation rule}

Using the triangulation in Fig. 14 and the central summation rule, only 651 repatoms and 911 sampling atoms are used for this test case. This corresponds to the use of $14.0 \%$ of all atoms as repatoms and $19.6 \%$ of all atoms as sampling atoms.

Comparing the left image and the central image in Fig. 15, the vertical displacements in the coarse domain and fully resolved domain seem to be well predicted by the use of the central summation rule. At the transition of the coarse domain and the fully resolved domain however, discrepancies can be seen, as was the case for the previous test example.

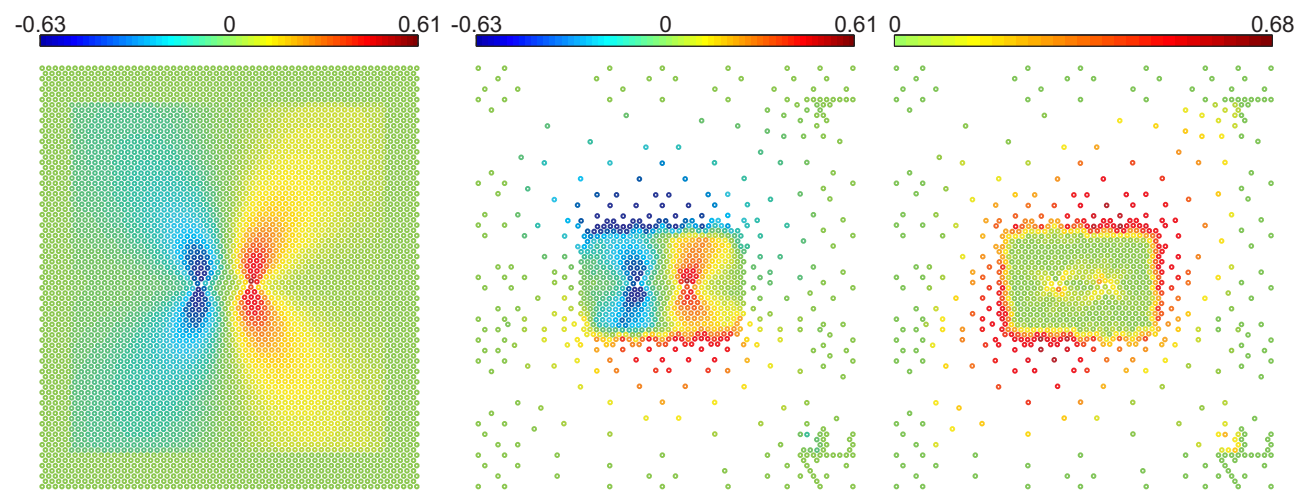

Figure 15: The solution predicted for the Lomer dipole with the direct computation (left) and with the QC computation using the central summation rule and the triangulation shown in Fig. 14 (center). The colors show the vertical ([ll $\left.\left.\begin{array}{ll}1 & 0\end{array}\right]\right)$ displacement components (in $\AA$ ). In the right image, the relative displacement, $u^{\text {rel }}$, based on Eq. (21), is shown in $\AA$..

To visualize the discrepancy between the displacement fields using the central summation rule and by the direct lattice computation, a relative displacement, $u^{r e l}$, is introduced as follows:

$$
u^{r e l}\left(\vec{X}^{i}\right)=\left\|\vec{u}^{q c}\left(\vec{X}^{i}\right)-\vec{u}^{d l c}\left(\vec{X}^{i}\right)\right\|,
$$


where $\vec{u}^{q c}$ is the displacement of atom $i$ predicted by the QC computation and $\vec{u}^{d l c}$ is the reference displacement of atom $i$. In the right image of Fig. 15 the relative displacement $u^{r e l}$ is shown.

It can be observed that the relative displacement is large in the coarse domain close to the fully resolved region (with a maximum of approximately $0.68 \AA$ ), although true ghost-forces (using the definition of Shenoy et al. (1999) and Miller and Tadmor (2002)) are not present in the central summation rule. If we regard the solution in the region of interest however, the relative displacement is significantly smaller. The average relative displacement of the ten atoms closest to the two dislocation cores is for instance $0.15 \AA$.

The error in this test case is significantly larger than for the previous test case in which a vacancy was considered. This can be attributed to two causes, although it is not possible to quantify which of these has the most influence. First, the dislocations introduce significantly larger displacement fluctuations than a single vacancy. Moreover, the use of the EAM ensures a higher degree of nonlocality compared to the use of the Lennard-Jones potential. This leads to an increased influence of the displacement fluctuations around the fully resolved region on the solution in the center of the fully resolved region.

\subsection{Vacancy in a three-dimensional crystal using the Lennard-Jones poten- tial}

The use of the central summation rule for three-dimensional atomistic crystals is demonstrated by modeling a vacancy in a three-dimensional cubic FCC crystal. The considered crystal contains 1,074,344 atoms $(64 \times 64 \times 64$ unit cells). Using the triangulation of Fig. 16 which includes a fully resolved region of $8 \times 8 \times 8$ unit cells, only 8,732 repatoms and 55,744 sampling atoms need to be used. The computational cost in terms of DOFs (number of repatoms) and effort to construct the governing equations (sampling atoms) is thus reduced to $0.8 \%$ and $5.1 \%$ respectively of the full atomistic analysis.

The boundary conditions are equivalent with those in the two-dimensional examples. The FCC crystal is uniaxially strained to $0.5 \%$ in $\left[\begin{array}{lll}1 & 0 & 0\end{array}\right]$ direction. Since the outer tetrahedra contain a significant number (approximately 85) of atoms that are sampled by the central sampling atom, the ratio between the number of atoms represented by the central sampling atom and the four discrete sampling atoms (i.e. the repatoms) in one tetrahedron is large. Therefore, the edge effects are expected to be small and no fully constrained band of tetrahedra is necessary (cf. the slim triangles that surround the twodimensional models). This saves computational efforts. 


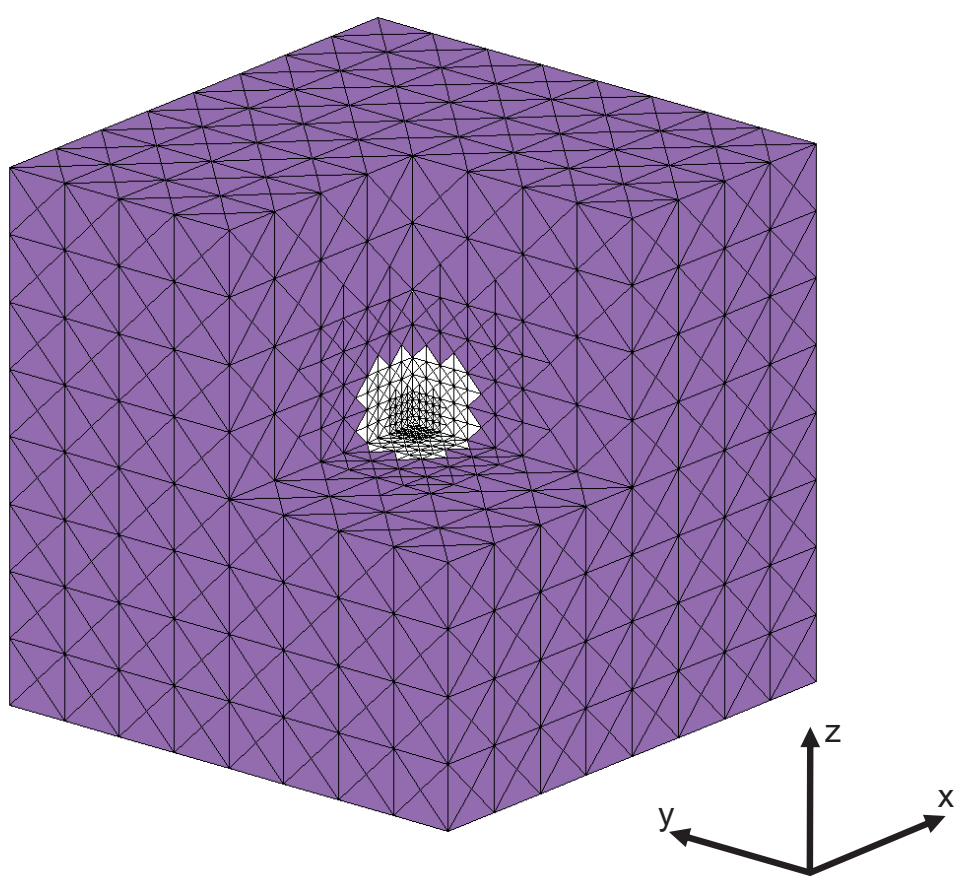

Figure 16: Part $\left(\frac{7}{8}\right)$ of the triangulation for the three-dimensional cubic FCC crystal including a vacancy in the center. The axes correspond to the $\langle 100\rangle$ orientations of the FCC crystal. The purple tetrahedra contain central sampling atoms. The white tetrahedra contain only discrete sampling atoms because all atoms in these tetrahedra are located on top of the repatoms (in the fully resolved regions) or on top of tetrahedron edges and faces (in the coarse domain around the fully resolved region).

To investigate the influence of the vacancy, relative displacements of the repatoms are computed based on the the predicted displacement vectors ac-

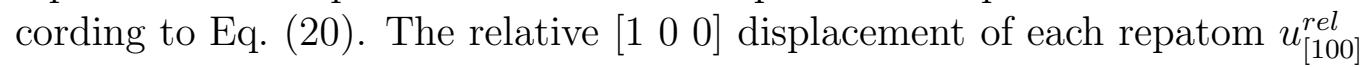
is the component of the predicted displacement vector in $\left[\begin{array}{lll}1 & 0 & 0\end{array}\right]$ direction relative to the displacement component in $\left[\begin{array}{lll}1 & 0 & 0\end{array}\right]$ direction for a FCC crystal without a vacancy subjected to the same uniaxial strain.

\subsubsection{Solution predicted using the central summation rule}

The relative $\left[\begin{array}{lll}1 & 0 & 0\end{array}\right]$ displacements of the repatoms are presented in Fig. 17. A number of observations can be made based on Fig. 17 that are distinct from the two-dimensional results, revealing intrinsic differences in three- 
dimensional crystals.

Eight atoms around the vacancy show distinct relative $\left[\begin{array}{lll}1 & 0 & 0\end{array}\right]$ displacements with a magnitude of $0.00149 r_{0}$. This effect decays away from the vacancy over three lattice spacings. The four atoms next to the vacancy in the face given by the $\left[\begin{array}{lll}1 & 0 & 0\end{array}\right]$ normal direction show no distinct relative $[1$ 0 0] displacements. This is caused by the fact that these four neighboring atoms of the vacancy are located in the face given by the normal direction that corresponds to the loading direction.

The most striking difference with the two-dimensional results is that the relative $\left[\begin{array}{lll}1 & 0 & 0\end{array}\right]$ displacements of the three-dimensional crystal reveal that the neighboring atoms of the vacancy are moving towards the location of the vacancy whereas the neighboring atoms of the vacancy in the two-dimensional crystal move away from the vacancy. This is caused by the differences in twodimensional and three-dimensional configurations.

The quality of the summation rule for this three-dimensional example is relatively difficult to evaluate since the semi-QC model in which $S=N$ is computationally too expensive. However, the accuracy of the relative $\left[\begin{array}{ll}1 & 0\end{array}\right.$ 0] displacements of the repatoms in the fully resolved regions appears to be sufficient since no significant fluctuations can be observed in the fully resolved domain as in a number of two-dimensional results, e.g. in the left image of Fig. 12. Apparently, the size of the fully resolved domain $(8 \times 8 \times 8$ unit cells) is sufficiently large to obtain an adequate solution for a vacancy in the considered lattice.

As in the two-dimensional results (see Fig. 12), some fluctuations occur in the small tetrahedra around the fully resolved domain (see the left images in Fig. 17). However, because for the FCC crystal with the triangulation shown in Fig. 16 a relatively large number of small tetrahedra occur that have all their atoms on tetrahedron edges and faces (see the white triangles in Fig. 16), a relatively large number of atoms around the fully resolved region are selected as discrete sampling atoms compared to the two-dimensional crystal. For this reason the fluctuations induced in the transition region between the coarse and full resolved domain are smaller compared to the fluctuations in the two-dimensional results. 


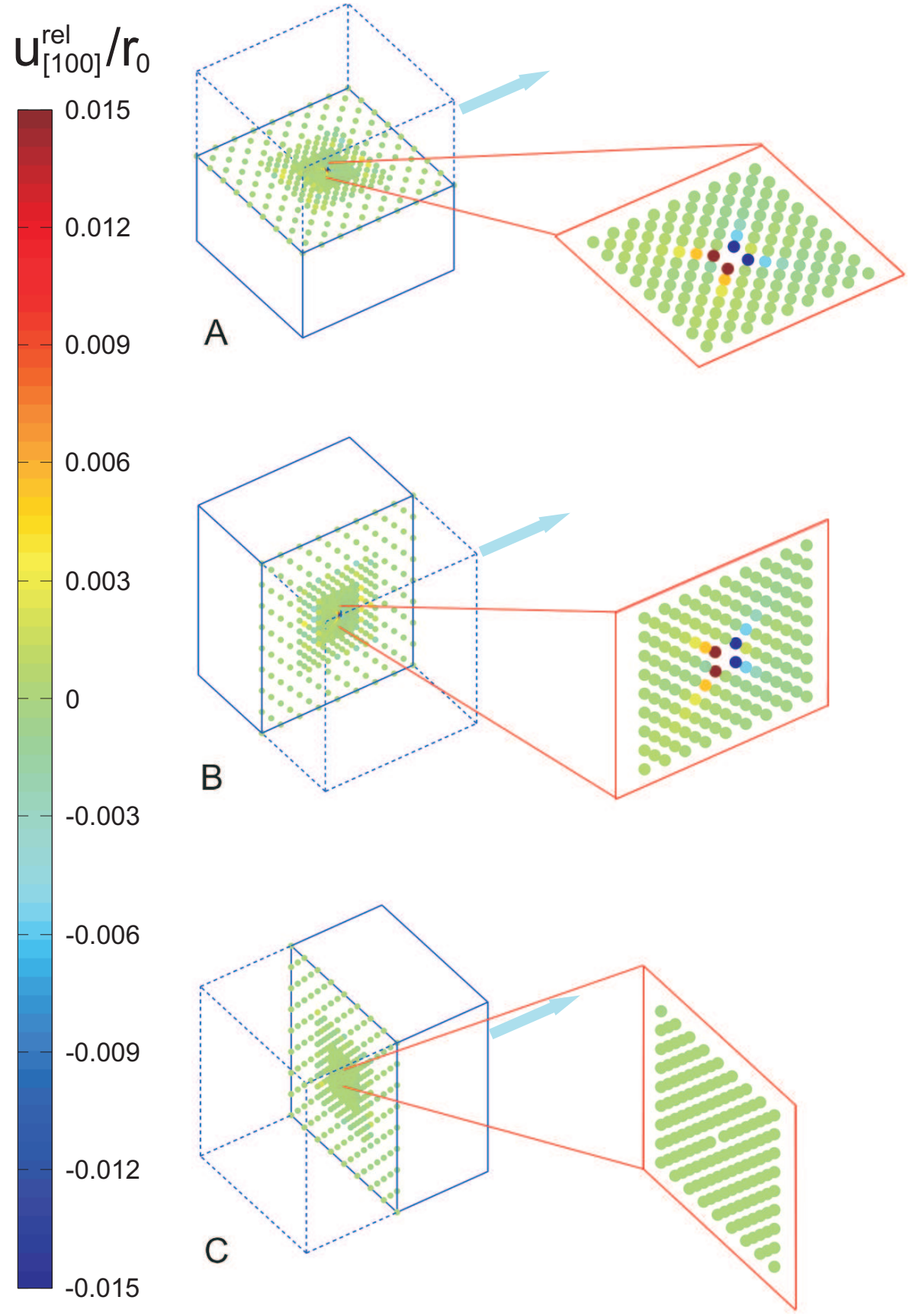

Figure 17: The relative displacement components scaled by $r_{0}$ in $\left[\begin{array}{lll}1 & 0 & 0\end{array}\right]$ direction of the repatoms in three cross-sectional faces with [ $\left[\begin{array}{lll}0 & 0 & 1\end{array}\right]$ normal (image A), [ $\left.\begin{array}{lll}0 & 1 & 0\end{array}\right]$ normal (image B) and $\left[\begin{array}{lll}1 & 0 & 0\end{array}\right]$ normal (image C) through the center of the $\left.<100\right\rangle$ oriented FCC crystal. Zooms around the vacancy are presented in the right images. The arrows indicate the loading in $\left[\begin{array}{lll}1 & 0 & 0\end{array}\right]$ direction. 


\section{Conclusion}

The aim of this paper was to develop a new summation rule for the quasicontinuum method (Tadmor et al., 1996a) that can efficiently deal with atomistic crystals while avoiding an internal interface. The proposed central summation rule is based on a clear understanding of the relation between the potential energy and the interpolation, as the summation rule presented by Beex et al. (2011) for structural lattice models. In contrast to the summation rule of Beex et al. (2011), which leads to a large number of sampling atoms if applied to atomistic crystals (especially in three dimensions), the number of sampling atoms selected in the central summation rule is small to gain computational efficiency. Consequently, the potential energy is estimated instead of determined exactly. The central summation rule significantly reduces the computational cost of full atomistic computations as a result of the use of the repatoms as sampling atoms in combination with one extra sampling atom in the center of coarse triangles. The focus of the central summation rule is thus on the center of the triangles and tetrahedra, which is more natural compared to clusters of sampling atoms around the repatoms (Knap and Ortiz, 2001; Eidel and Stukowski, 2009). The algorithm for the summation rule deals with triangles in the coarse domains and fully resolved domains in a unified way and is therefore simple to implement.

The energy-based central summation rule uses a nonlocal site-energy computation in the coarse domain as well as in the fully resolved domain. As a result, no ghost-forces occur between both domains, similar to the cluster summation rules (Knap and Ortiz, 2001; Eidel and Stukowski, 2009). This ensures that moving and growing/shrinking fully resolved regions can easily be included since no internal interface procedures have to be implemented and updated. Adaptivity can therefore more straightforwardly be implemented in a quasicontinuum framework and this is one of the focus points of future research.

In one of the numerical examples a vacancy is considered in a two-dimensional closely packed atomistic lattice, in which the atomistic interactions are governed by Lennard-Jones potentials. A comparison between the results predicted using the central summation rule and those predicted using the energy-based cluster summation rule (Eidel and Stukowski, 2009) shows that the central summation rule is not only more efficient, but also significantly more accurate. Results predicted for two dislocation cores using the 
embedded-atom-method are not as accurate as for a single vacancy, which is caused by the fact that two dislocation cores introduce more pronounced displacement fluctuations than a single vacancy and by the fact that the embedded-atom-method has a higher degree of nonlocality than straightforward Lennard-Jones interactions. The applicability of the central summation rule in three-dimensions is shown as well, but its results could not be compared to the reference solution, which was computationally too expensive to compute.

The central summation rule has in common with the local-nonlocal summation rule (Tadmor et al., 1996a; Shenoy et al., 1999) that in the limit of large interpolation triangles and tetrahedra it equals the local summation rule using the Cauchy-Born rule. This is a result of the fact that in large interpolation triangles all neighboring atoms of the central sampling atoms are located in the same interpolation triangles as the central sampling atoms. Furthermore, since the weight factors $\left(w_{i}\right)$ of the central sampling atoms are large in large triangles compared to the weight factors of the discrete sampling atoms $\left(w_{i}=1\right)$, the discrete (corner) sampling atoms' contribution becomes negligible. A natural and smooth transition is thus obtained from a fully nonlocal method in refined regions to an almost local method in coarse regions.

The central summation rule combines most advantageous characteristics of the two main classes of summation rules:

- no internal interface occurs (as in the cluster summation rules),

- it uses almost as few sampling atoms as the local-nonlocal rules,

- it is more accurate than the cluster summation rules (compared to the local-nonlocal summation rules).

A disadvantage is that, as in the cluster summation rules, to ensure that no internal interface occurs, the site-energies of all sampling atoms must be computed nonlocally and thus relatively large lookup tables are necessary. However, this seems to be inevitable for summation rules in which internal interfaces are to be eliminated.

\section{Acknowledgements}

This research is supported by the Dutch Technology Foundation STW, which is the applied science division of NWO, and the Technology Programme 
of the Ministry of Economic Affairs under Project Nr. 10104.

\section{References}

Beex, L.A.A., Peerlings, R.H.J., Geers, M.G.D., 2011, A quasicontinuum methodology for multiscale analyses of discrete microstructures, International Journal for Numerical Methods in Engineering, Vol. 87, 701-718.

Curtin, W.A, Miller, R.E., 2003, Atomistics/continuum coupling in computational materials science, Modelling and Simulation in Materials Science and Engineering, Vol. 11, 33-68.

Dobson, M., Elliott, R.S., Luskin, M., Tadmor, E.B., 2007, A multilattice quasicontinuum for phase transformation materials: cascading cauchyborn kinematics, Journal of Computer-Aided Materials Design, Vol. 14, 219-237.

Eidel, B., Stukowski, A., 2009, A variational form of the quasicontinuum method based on energy sampling in clusters, Journal of the Mechanics and Physics of Solids, Vol. 57, 87-108.

Gavini, V., Bhattacharya, K., Ortiz, M., 2007, Quasicontinuum orbital-free density functional theory: a route to multi-million atom non-periodic DFT calculation, Journal of the Mechanics and Physics of Solids, Vol. 55, 697718.

Gunzburger, M., Zhang, Y., 2010, A quadrature-rule type approximation to the quasi-continuum method, Multiscale Modeling and Simulation, Vol. 8, 571-590.

Hayes, R.L., Fago, M., Ortiz, M., Carter, E.A., 2005, Density-functionaltheory-based local quasicontinuum method: prediction of dislocation nucleation, Physical Review B, Vol. 90, 100102.

Hirth, J.P., Lothe, J., 1996, Theory of dislocations, Krieger Publishing, Malabar, USA.

Iyer, M., Gavini, V., 2011, A field theoretical approach to the quasicontinuum method, Journal of the Mechanics and Physics of Solids, Vol. 59, 1506-1535. 
Knap, J., Ortiz, M., 2001, An analysis of the quasicontinuum method, Journal of the Mechanics and Physics of Solids, Vol. 49, 1899-1923.

Knap, J., Ortiz, M., 2003, Effect of indenter radius size on $\mathrm{Au}(001)$ nanoindentation, Physical Review Letters, Vol. 90, 226102.

Kulkarni, Y., Knap, J., Ortiz, M., 2008, A variational approach to coarse graining of equilibrium and non-equilibrium atomistic description at finite temperature, Journal of the Mechanics and Physics of Solids, Vol. 56, 1417-1449.

Kwon, S., Lee, Y., Park, J.Y., Sohn, D., Lim, J.H., Im, S., 2009, An efficient three-dimensional adaptive quasicontinuum method using variable-node elements, Journal of Computational Physics, Vol. 228, 4789-4810.

Lennard-Jones, J.E., 1937, The equation of state of gases and critical phenomena, Physica IV, Vol. 10, 941-956.

Luskin, M., Ortner, C., 2008, An analysis of node-based cluster summation rules in the quasicontinuum method, SIAM Journal on Numerical Analysis, Vol. 47, 3070-3086.

Marian, J., Knap, J., Campbell, G.H., 2008, A quasicontinuum study of nanovoid collapse under uniaxial loading in Ta, Acta Materiala, Vol. 56, 2389-2399.

Marian, J., Venturini, G., Hansen, B.L., Knap, J., Ortiz, M., Campbell, G.H., 2010, Finite-temperature extensions of the quasicontinuum method using Langevin dynamics: entropy losses and analysis of errors, Modelling and Simulations in Material Science and Engineering, Vol. 18, 015003.

Miller, R., Ortiz, M., Phillips, R., Shenoy, V., Tadmor, E.B., 1998, Quasicontinuum models of fracture and plasticity, Engineering Fracture Mechanics, Vol. 61, 427-444.

Miller, R.E., Tadmor, E.B., 2002, The quasicontinuum method: overview, applications and current directions, Journal of Computer-Aided Materials Design, Vol. 9, 203-239.

Miller, R.E., Rodney, D., 2008, On the nonlocal nature of dislocation nucleation during nanoindentation, Journal of the Mechanics and Physics of Solids, Vol. 56, 1203-1223. 
Miller, R.E., Tadmor, E.B., 2009, A unified framework and performance benchmark of fourteen multiscale atomistic/continuum coupling methods, Modelling and Simulation in Materials Science and Engineering, Vol. 17, 053001 .

Mitev, P., Evangelakis, G.A., Kaxiras, E., 2006, Embedded atom method potentials employing a faithful density representation, Modelling and Simulation in Materials Science and Engineering, Vol. 14, 721-731.

Park, J.Y., Im, S., 2008, Adaptive nonlocal quasicontinuum for deformations of curved crystalline structures, Physical Review B, Vol. 77, 184109.

Park, J.Y., Park, C.H., Park, J.S., Kong. K., Chang, H., Im, S., 2010, Multiscale computation for carbon nanotubes based on a hybrid QC/QM (quantum mechanical and quasicontinuum) approach, Journal of the Mechanics and Physics of Solids, Vol. 77, 184109.

Picu, R.C., 2000, Atomistic-continuum simulation of nano-indentation in molybdenum, Journal of Computed-Aided Materials Design, Vol. 7, 7787.

Shenoy, V.B., Miller, R., Tadmor, E.B., Rodney, D., Phillips, R., Ortiz, M., 1999, An adaptive finite element approach to atomistic scale mechanics-the quasicontinuum method, Journal of the Mechanics and Physics of Solids, Vol. 47, 611-642.

Shimokawa, T., Mortensen, J.J., Schiøtz, J., Jacobsen, K.W., 2004, Matching conditions in the quasicontinuum method: Removal of the error introduced at the interface between the coarse-grained and fully atomistic region, Physical Review B, Vol. 69, 214104.

Tadmor, E.B., Phillips, R., Ortiz, M., 1996, Mixed atomistics and continuum models of deformation in solids, Langmuir, Vol. 12, 4529-4534.

Tadmor, E.B., Ortiz, M., Phillips, R., 1996, Quasicontinuum analysis of defects in solids, Philosophical Magazine A, Vol. 73, 1529-1563.

Tadmor, E.B., Miller, R., Phillips, R., Ortiz, M., 1999, Nanoindentation and incipient plasticity, Journal of Materials Research, Vol. 14, 2233-2250. 
Zhang, Y., Gunzburger, M., 2010, Quadrature-rule type approximations to the quasicontinuum method for long-range interatomic interactions, Computer Methods in Applied Mechanics and Engineering, Vol. 199, 648-659. 